\title{
A dynamic taxi traffic assignment model: A two-level continuum transportation system approach
}

by

\author{
Jiancheng Long \\ Professor \\ School of Automation and Transportation Engineering \\ Hefei University of Technology \\ Hefei 230009, China \\ Tel: +86-551-63831101 \\ E-mail: jianchenglong@hfut.edu.cn
}

\section{W.Y. Szeto}

Associate Professor

Department of Civil Engineering

The University of Hong Kong

Pokfulam Road, Hong Kong

Tel: +852-28578552

Email: ceszeto@hku.hk

\section{Jie Du}

Postdoctoral Fellow

Department of Mathematics

The Chinese University of Hong Kong

Shatin, NT, Hong Kong

Tel: +852-55121471

Email: jdu@math.cuhk.edu.hk

\section{R.C.P. Wong}

Lecturer

Department of Civil Engineering

The University of Hong Kong

Pokfulam Road, Hong Kong

Tel: +852-39175156

Email: cpwryan@hku.hk 


\begin{abstract}
This paper proposes a two-level continuum transportation system approach to modeling a dynamic taxi traffic assignment (DTTA) problem in a dense network with real-time traffic information provision and three types of vehicles, including private cars, occupied taxis, and vacant taxis. The proposed approach treats the dense network as a continuum in the first level, in which private car and occupied taxi drivers are free to choose their paths in a two-dimensional continuous space. The proposed approach also divides the modeling region into many identical squares to form a cell-based network in the second level, in which the cells are classified into two categories: target cell with an acceptable expected rate of return (EROR) to vacant taxi drivers and non-target cell with an unacceptable EROR. The EROR associated with a cell is the ratio of the cumulative expected profit of a taxi driver who successfully picks up a customer during the customer search that starts from that cell to the sum of expected search time for this customer and expected occupied travel time to serve this customer. Based on the cell-based network, we develop a cell-based intervening opportunity model to capture that fact that vacant taxi drivers can meet a customer on the way to their destination zones and to estimate the EROR. Each vacant taxi driver has a mixed strategy to determine his/her customer-search direction according to the EROR: Each vacant taxi driver in a target cell selects its neighbor cells with maximum EROR, and each vacant taxi driver in a non-target cell selects the travel time-based shortest path to his/her target cell. Meanwhile, each private car driver chooses the path that minimizes his/her own generalized travel cost, and each occupied taxi driver chooses the path that minimizes his/her customer's generalized in-vehicle travel cost. In our model, traffic density in the system is governed by the conservation law (CL), and the flow directions of different vehicles are determined by the path-choice strategies of their drivers, which are captured by Hamilton-Jacobi (HJ) equations. Both the proposed CL and HJ equations can be solved by the Lax-Friedrichs scheme, which forms the backbone of the developed solution algorithm. Finally, numerical examples and a case study are used to demonstrate the properties of the model, the performance of the solution algorithm, and the value of using our methodology for estimating network performance.
\end{abstract}

Keywords: Continuum model, taxi customer-search, intervening opportunity model, conservation law, Hamilton-Jacobi equation, reactive dynamic user optimal. 


\section{Introduction}

As an important transportation mode, taxis offer speedy, comfortable, and direct transportation services. However, they tend to circulate around a city to search for customers and can consequently use up significant amount of road capacity and worsen traffic congestion and roadside air quality. To tackle these problems, advanced technologies (e.g., Horn, 2002; Lee and Cheng, 2008; Conway et al., 2012; da Costa and de Neufville, 2012; Miwa et al., 2013; Jung et al., 2014) are often used, and taxi regulation policies (e.g., De Vany, 1975; Schroeter, 1983; Arnott, 1996; Cairns and Liston-Heyes, 1996; Yang et al., 2000, 2002, 2005, 2010; Fernández et al., 2006; Moore and Balaker, 2006; Wong et al., 2014b) are established. The effectiveness of these methods can be evaluated by a taxi traffic assignment model.

Taxi traffic assignment aims to predict taxi traffic flow on each link or in each region, and can be used for both offline taxi regulation policy evaluation and real-time taxi operation and management. Taxi traffic assignment is an extension of traditional traffic assignment problems (TAPs), which have been widely studied for a long time and can be classified into two categories: static TAPs (e.g., Beckmann et al., 1956; Daganzo and Sheffi, 1977; Bar-Gera, 2002; Nie, 2010) and dynamic traffic assignment (DTA) problems (e.g., Yagar, 1971; Merchant and Nemhauser, 1978a,b; Friesz et al., 1989; Ran et al., 1993; Huang and Lam, 2002; Lo and Szeto, 2002; Ban et al., 2008; Long et al., 2013, 2015, 2016; Han et al., 2015). As the temporal variation of flow and cost are not considered in the static models, they cannot be used to model travelers' departure/arrival time choices or dynamic traffic management and control. DTA can address these problems and has received much attention in recent decades (e.g., Merchant and Nemhauser, 1978a,b; Friesz et al., 1989; Ran et al., 1993; Lo and Szeto, 2002; Long et al., 2013; Du et al., 2013, 2015; Jiang et al, 2017). Different from the fast development of TAP models, the development of taxi traffic assignment models is still in its early stage. Most existing taxi traffic assignment models are static (e.g., Yang and Wong, 1998; Wong et al., 2008; Yang et al., 2010). Therefore, they cannot capture the time-varying customer demand and the situation that taxi drivers change their customer search choices en-route when they receive real-time traffic and customer demand information regularly. Consequently, the taxi customer-search efficiency and the congestion impacts caused by taxi cruising and circulation during the studied horizon cannot be accurately evaluated by these models.

In the literature, there are two approaches to modeling TAPs: discrete (e.g., Friesz et al., 1993; Huang and Lam, 2002; Lo and Szeto, 2002; Lim and Heydecker, 2005; Long et al., 2013, 2015, 2016; Jiang et al., 2016) and continuum (e.g., Hoogendoorn and Bovy, 2004; Jiang et al., 2011, 2017; Du et al., 2013, 2015). The discrete modeling approach assumes that road links are separated but connected by nodes, and traffic demands are concentrated at hypothetical zone centroids. Discrete traffic equilibrium assignment problems are usually formulated as certain well-known mathematical problems, such as mathematical programming problems (e.g., Merchant and Nemhauser, 1978a,b; Carey, 1987; Carey and Subrahmanian, 2000; Ziliaskopoulos, 2000; Nie, 
2011; Waller et al., 2013), optimal control problems (e.g., Friesz et al., 1989; Ran et al., 1993; Ma et al., 2014), variational inequality (VI) problems (e.g., Friesz et al., 1993; Ran and Boyce, 1996; Huang and Lam, 2002; Han, 2003; Long et al., 2013), nonlinear complementarity problems (NCP) (e.g., Wie et al., 2002; Ban et al., 2008), fixed-point problems (e.g., Smith, 1993; Lim and Heydecker, 2005; Szeto et al., 2011; Long et al., 2015), etc. The solution properties such as the existence and uniqueness of solution to the analytical DTA models can be determined beforehand and the existing solution algorithms can be used to solve them. However, it is challenging to model a large-scale congested urban traffic network with a large number of highways, intersections, interchanges, and severe congestion with queues and their spillbacks.

The continuum approach approximates a dense network as a continuum in which travelers are free to choose their paths in a two-dimensional continuous space. This allows that the characteristics of a network, such as its flow intensity, demand, and travel cost, can be represented by smooth mathematical functions (Vaughan, 1987). Hence, fewer data are required for the model setup process, and the problem size can be reduced for large dense transportation networks. This can potentially save computational time and memory. This approach also includes a special case where a single corridor is modelled as a continuum (e.g., Wang and $\mathrm{Du}, 2013$; Du and Wang, 2014). The continuum modeling approach has been widely applied to model various highly dense transportation systems (e.g., Wong et al., 1998; Yang and Wong, 2000; Ho et al., 2006, 2013; Hoogendoorn and Bovy, 2004; Jiang et al., 2011; Du et al., 2013, 2015). Early studies use static continuum equilibrium models to determine facility locations and perform policy (e.g., Yang and Wong, 2000; Ho et al., 2006), environmental (e.g., Yin et al., 2013) and socio-economic analyses (e.g., Ho et al., 2013). These static models can predict a long-term traffic state for transportation planning and network design, but should only be used when traffic flow variation is small. To overcome this shortcoming, continuum static equilibrium models have been extended to continuum DTA models for pedestrian flow (e.g., Xia et al., 2008; Huang et al., 2009; Jiang et al., 2012) and urban road traffic flow (e.g., Hoogendoorn and Bovy, 2004; Jiang et al., 2011; Du et al., 2013, 2015; Wang and $\mathrm{Du}, 2016$ ). However, to the best of our knowledge, continuum DTA models for taxi traffic flow has not been proposed in the literature.

The taxi route choice principle is a fundamental component of taxi traffic assignment models. It depicts how taxi drivers select their routes. The routing strategy of a taxi driver depends on taxi occupation status. If a taxi is occupied by customers, then the minimum cost path is usually assumed to be used (e.g., Yang and Wong, 1998; Yang et al., 2002; Wong et al., 2001, 2008). If not, the main principle used to depict the route choice of a vacant taxi driver varies from study to study, and includes minimizing the expected search time for customers (Hu et al., 2012), maximizing weighted expected profit minus search cost (Wong et al., 2003), maximizing profit per unit time (Yang et al., 2010), etc. However, all of these route choice principles ignore the fact that vacant taxi drivers can meet a customer on the way to their destination zones. To capture this intervening opportunity feature, Wong et al. (2015b) considered the sequential customer-search decisions of 
vacant taxi drivers on finding customers at intermediate zones while heading to their designated zones. However, the behavior model of Wong et al. (2015b) ignores the fact that as vacant taxi drivers search for customers, the probability of successfully meeting a taxi customer along the way increases. Wong et al. (2014b) proposed the concept of the cumulative probability of success of being hired, and developed a logit-opportunity modeling approach to modeling the taxi customer-search behavior in a cell-based network. However, the route choice principle of vacant taxi drivers used by Wong et al. (2014b) is maximizing the cumulative probability of success, and ignores the expected profit of taxi drivers, which is always their concern. Both profitability and the cumulative probability of success of being hired should be considered in the search choice principle. Moreover, as vacant taxi drivers do not always have complete and very accurate knowledge on their profitability of their following occupied trips, they may not be rational but instead boundedly rational, which is consistent with our frequent observations that vacant taxis are normally not too concentrating on very few expected profitable locations or narrow regions to search for their next customer. The boundedly rational consideration should also be captured in the search choice principle of vacant taxi drivers.

In a city, the common major source of road congestion is private cars, and traffic congestion significantly influences the route choice of taxi drivers. Meanwhile, taxis can use up significant amount of road capacity (for example in Hong Kong) and can worsen traffic congestion due to their cruising to search for customers, and hence taxis influence also the route choice of car drivers. Therefore, private cars and taxis have interactions on each other, and should be simultaneously considered in a taxi traffic assignment model.

In this paper, we propose a two-level continuum transportation system approach to modeling a dynamic taxi traffic assignment (DTTA) problem in a dense network with real-time traffic information. This approach treats the dense network as a continuum in the first level, in which private car and occupied taxi drivers are free to choose their paths in a two-dimensional continuous space. This approach also divides the modeling region into many identical squares to form a cell-based network for vacant taxi drivers to search for customers in the second level, in which the cells are classified into two categories: target cell with an acceptable expected rate of return (EROR) to vacant taxi drivers and non-target cell with an unacceptable EROR. The EROR associated with a cell is the ratio of the cumulative expected profit of a taxi driver who successfully picks up a customer during the customer search that starts from that cell to the sum of expected search time for this customer and expected occupied travel time to serve this customer. In the modeling region, there are three types of vehicles: private cars, vacant taxis, and occupied taxis. Different types of drivers are assumed to have their own path choice strategy_Each private car driver chooses the path that minimizes his/her own generalized travel cost; each occupied taxi driver chooses the path that minimizes his/her customer's generalized in-vehicle travel cost, and each vacant taxi driver has a mixed strategy to determine his/her customer-search direction according to the EROR: Each vacant taxi driver in a target cell selects its neighbor 
cells with the maximum EROR, and each vacant taxi driver in a non-target cell selects travel time-based shortest path to the target cell. Hamilton Jacobi $(\mathrm{HJ})$ equations are proposed to compute the minimum generalized travel costs of private car drivers and customers' generalized in-vehicle travel cost. Based on the cell-based network, an intervening opportunity sub-model is developed to capture the cumulative probability of successfully meeting a customer in each direction of customer-search and the profitability concern of vacant taxi drivers, and to estimate the EROR of vacant taxi drivers. In our proposed model, the traffic density of each vehicle type is governed by the conservation law (CL), in which the flow direction is determined by the proposed path-choice strategies and HJ equations for all types of vehicles. This implies that our proposed model captures the interaction between private cars and taxis and their contributions to traffic congestion.

To solve our model numerically, we develop an algorithm that uses the Lax-Friedrichs scheme to solve the proposed HJ equations and CLs. The Lax-Friedrichs scheme is a conservative monotone scheme with desirable properties such as the maximum principle and total variation diminishing (TVD). Finally, numerical examples and a case study are used to demonstrate the properties of the model, the performance of the solution algorithm, and the importance of using our methodology for estimating network performance more accurately.

In terms of methodology, our proposed model has four main differences compared with the existing taxi traffic assignment models or existing continuum models.

First, we propose a different search choice principle to describe the path choice strategy of vacant taxi drivers compared with those in the taxi assignment literature. In our paper, we consider both the concept of the cumulative probability of success of being hired and the expected profit in the path choice strategy of vacant taxi drivers. Moreover, compared with the literature, we further introduce the concept of tolerance in the search choice principle to take into an account that vacant taxi drivers are boundedly rational.

Second, the analytical taxi assignment models in the literature are static, but we develop a DTTA model. Our model can capture time-varying customer demand and allow examining the impacts of regular real-time traffic and customer demand information update and traffic congestion on the path search strategy of vacant taxi drivers more accurately. This modeling approach can lead to the estimation of network performance more accurately. This is particularly important in many urban cities including Hong Kong where taxi cruising significantly contributes to road congestion.

Third, the taxi traffic assignment models in the literature are developed based on graph theory with a network formed by links and nodes. Our model is developed based on a two-level continuum approach and is more computationally efficient than the models developed by the traditional graph theory approach. This efficiency is very important for online traffic management and control applications, because new results have to be computed quickly to allow making effective management and control decisions after traffic information is updated each time. 
Fourth, our continuum model has two levels, and is different from the single level continuum models only for private cars.

The main contributions made in our research are as follows.

First, we propose a dynamic taxi assignment model for continuum transportation systems for online applications. It takes both congestion and traffic information update into account. To the best of our knowledge, it is the first analytical DTTA model that incorporates both the intervening opportunity concept and profitability consideration to model local customer search and considers a dense taxi traffic network as a two-level continuum system.

Second, we introduce a new concept—EROR, develop a novel intervening opportunity model to depict the local customer-search of vacant taxi drivers, and propose a new tolerance-based path choice strategy of vacant taxi drivers.

Third, this paper illustrates the properties of the model. In particular, this paper shows the effects of congestion and the duration between each traffic information update on the taxi customer-search efficiency as measured by customer waiting time. We also show that taxi and private cars interact with each other.

The remainder of this paper is organized as follows. The description of the dynamic taxi assignment problem in a continuum polycentric city is given in the next section. In Section 3, the dynamic taxi assignment problem is formulated. The solution algorithm for the proposed dynamic taxi assignment model is presented in Section 4. In Section 5, numerical examples and a case study are provided. Finally, our conclusions are drawn in Section 6.

\section{Problem description}

As shown in Fig. 1, we consider a polycentric urban region with $\underline{M}$ compact CBDs (or destinations in general). Let $M=\{1,2, \cdots, \underline{M}\}$ be the set of CBDs. In this region, the road network is very dense and can be viewed as a two-dimension continuum. Let $\Omega\left(\right.$ in $\mathrm{km}^{2}$ ) and $\Gamma$ (in $\left.\mathrm{km}\right)$, respectively, be the modeled urban region (i.e., the modeling domain, which excludes the CBDs and obstructions) and the boundary of the modeling domain. Let $\Gamma^{0}$ (in $\mathrm{km}$ ) be the outer boundary of $\Omega, \Gamma_{c}^{m}$ (in $\mathrm{km}$ ) be the boundary of the $m$-th compact $\mathrm{CBD}$, and $\Gamma_{o}^{n}$ (in $\mathrm{km}$ ) be the boundary of the $n$-th $(n=1,2, \ldots, N)$ obstruction, such as a lake, a park, or an undeveloped area where traffic is not allowed to enter or leave. Hence, the boundary of $\Omega$ is $\Gamma=\Gamma^{0} \cup \Gamma_{c}^{m} \cup \Gamma_{o}^{n}$. It is assumed that travelers' homes are continuously located over $(x, y) \in \Omega$.

Travelers go from their homes to their designated CBDs to work by either driving or taxi. Hence, there are three types of vehicles in the modeling region during the studied morning period: (1) private cars, (2) occupied taxis, and (3) vacant taxis. Based on vehicles' destination, there are $\underline{M}$ types of private cars, and $\underline{M}$ types of occupied taxis, and one type of vacant taxi. Let $K=\{0,1,2, \cdots, 2 \underline{M}\}$ represent the set of types of vehicles in the region. Let $K_{0}=\{0\}, K_{1}=\{1,2, \cdots, \underline{M}\}$, and $K_{2}=\{\underline{M}+1, \underline{M}+2, \cdots, 2 \underline{M}\}$, 
respectively, represent the set of types of vacant taxis, the set of types of occupied taxis, and the set of types of private cars.

We assume that the travel demand of both cars and taxi customers of the morning commute is time-dependent, fixed, and given, and hence our model described later does not include a choice between car and taxi from an origin to a destination. We also assume that all vehicles have real-time traffic information from a radio broadcasting service or a route guidance system. The traffic information is updated regularly, and let $\delta$ be the length of an information update interval. Based on the information update interval, the time period $T$ can be discretized into a finite set of time intervals $\Lambda=\{\tau=1,2, \cdots, \underline{\Lambda}\}$, and we have $T=\bigcup_{\tau \in \Lambda} T_{\tau}$, where $T_{\tau}$ is the time period of interval $\tau$. Let $\bar{T}$ and $\bar{T}_{\tau}$ be the end time of studied period and the end time of interval $\tau$, respectively. By definition, we have $T_{\tau}=\left(\bar{T}_{\tau-1}, \bar{T}_{\tau}\right]=((\tau-1) \delta, \tau \delta]$. The traffic information for vehicles' route choice during interval $\tau$ is based on the average traffic flow performance during interval $\tau-1$.

To formulate the customer-search behavior of vacant taxi drivers, we also divide the modeling region into many identical squares to form a cell-based network. Let $Z$ be the set of cells. Let $\Omega_{z}$ be the region of cell $z \in Z$ and $\Gamma_{z}$ be the boundary of region $\Omega_{z}$. The size of each cell can be adjusted subject to the required level of modeling accuracy.

The following notations are used throughout this paper:

\begin{tabular}{|l|l|}
\hline$\rho(x, y, t)$ & density of vehicles at location $(x, y)$ at time $t$ \\
\hline$\rho^{k}(x, y, t)$ & density of the $k$-th type of vehicle at location $(x, y)$ at time $t$ \\
\hline$\rho_{\tau}(x, y)$ & average vehicle density at location $(x, y)$ during time interval $\tau$ \\
\hline$\tilde{\rho}^{m}(x, y, t)$ & density of customers to the $m$-th CBD at location $(x, y)$ at time $t$ \\
\hline $\mathbf{v}(x, y, t)$ & $\begin{array}{l}\text { velocity vector at location }(x, y) \text { at time } t \text {, and } \mathbf{v}(x, y, t)=\left(v_{1}(x, y, t), v_{2}(x, y, t)\right) \\
\text { where } v_{1}(x, y, t) \text { and } v_{2}(x, y, t) \text { are velocities in the positive } x \text {-and } y \text {-directions at } \\
\text { time } t, \text { respectively }\end{array}$ \\
\hline$V(x, y, t)$ & $\begin{array}{l}\text { vehicle speed at location }(x, y) \text { at time } t, \text { which is the norm of the velocity vector, i.e., } \\
V(x, y, t)=|\mathbf{v}(x, y, t)|\end{array}$ \\
\hline$V_{\tau}(x, y)$ & average vehicle speed at location $(x, y)$ during time interval $\tau$ \\
\hline$V_{x, y}(\cdot)$ & $\begin{array}{l}\mathbf{f}^{k}(x, y, t)=\left(f^{k}(x, y, t), g^{k}(x, y, t)\right), \text { where } f^{k}(x, y, t) \text { and } g^{k}(x, y, t) \text { are the } \\
\text { flow in the positive } x \text { - and } y \text {-directions at time } t, \text { respectively }\end{array}$ \\
\hline $\mathbf{f}^{k}(x, y, t)$
\end{tabular}




\begin{tabular}{|c|c|}
\hline$q^{k}(x, y, t)$ & demand of the $k$-th type of vehicle at location $(x, y)$ at time $t$ \\
\hline $\bar{q}^{m}(x, y, t)$ & demand of private cars to the $m$-th CBD at location $(x, y)$ at time $t$ \\
\hline$\tilde{q}^{m}(x, y, t)$ & demand of customers to the $m$-th CBD at location $(x, y)$ at time $t$ \\
\hline$c_{\tau}(x, y)$ & $\begin{array}{l}\text { local travel cost per unit distance for private cars traveling at location }(x, y) \text { during } \\
\text { time interval } \tau\end{array}$ \\
\hline$t_{\tau}(x, y)$ & $\begin{array}{l}\text { local travel time per unit distance of travel incurred by all vehicles at location }(x, y) \\
\text { during time interval } \tau\end{array}$ \\
\hline$\varpi_{\tau}(x, y)$ & $\begin{array}{l}\text { local fare of a taxi ride per unit distance for occupied taxis traveling at location }(x, y) \\
\text { during time interval } \tau\end{array}$ \\
\hline$\mu_{\tau}(x, y)$ & $\begin{array}{l}\text { local generalized in-vehicle travel cost per unit distance of travel incurred by } \\
\text { customers at location }(x, y) \text { during time interval } \tau\end{array}$ \\
\hline$\phi_{\tau}^{k}(x, y)$ & $\begin{array}{l}\text { total travel cost (disutility) incurred by the } k \text {-th }\left(k \in K_{1} \cup K_{2}\right) \text { type of vehicle that } \\
\text { departs from location }(x, y) \text { during time interval } \tau \text { to its destination using the } \\
\text { constructed path-choice strategy }\end{array}$ \\
\hline $\mathbf{n}_{\tau}^{k}(x, y)$ & $\begin{array}{l}\text { flow direction of the } k \text {-th type of vehicle traveling at location }(x, y) \text { during time } \\
\text { interval } \tau\end{array}$ \\
\hline$\eta_{\tau}^{m}(x, y)$ & $\begin{array}{l}\text { travel time for a vacant taxi driver who picks up a customer to the } m \text {-th CBD at } \\
\text { location }(x, y) \text { during time interval } \tau\end{array}$ \\
\hline$P_{\tau}^{m}(x, y)$ & $\begin{array}{l}\text { profit for a taxi driver after picking up a customer to the } m \text {-th CBD at location }(x, y) \\
\text { during time interval } \tau\end{array}$ \\
\hline$P_{\tau}^{z}$ & $\begin{array}{l}\text { average profit of a taxi driver after successfully picking up a customer in cell } z \\
\text { during interval } \tau\end{array}$ \\
\hline$T_{\tau}^{z}$ & $\begin{array}{l}\text { average occupied travel time of a taxi driver after successfully picking up a customer } \\
\text { in cell } z \text { during interval } \tau\end{array}$ \\
\hline
\end{tabular}

\section{The dynamic taxi traffic assignment model}

\subsection{The path choice strategy of both occupied taxi and private car drivers}

\subsubsection{The formulations of the local generalized travel costs of private cars and occupied taxis}

The widely used Lighthill-Whitham-Richard (LWR) macroscopic modeling approach is adopted to formulate road traffic flow in the polycentric city (Huang et al., 2009 and Du et al., 2013). We assume the traffic flow follows a macroscopic fundamental diagram (MFD) (Daganzo and Geroliminis, 2008; Geroliminis and Daganzo, 2008; Geroliminis and Sun, 2011):

$$
V(x, y, t)=V_{x, y}(\rho(x, y, t)), \forall(x, y) \in \Omega, t \in T,
$$


The vehicle density at location $(x, y)$ at time $t$ can be expressed as follows:

$$
\rho(x, y, t)=\sum_{k \in K} \rho^{k}(x, y, t), \forall(x, y) \in \Omega, t \in T .
$$

The average vehicle density at location $(x, y)$ during time interval $\tau$ can be formulated as follows:

$$
\rho_{\tau}(x, y)=\frac{\int_{\bar{T}_{\tau-1}}^{\bar{T}_{\tau}} \rho(x, y, t) d t}{\bar{T}_{\tau}-\bar{T}_{\tau-1}}, \forall(x, y) \in \Omega, \tau \in \Lambda .
$$

Using the flow-density relationship (1), the average vehicle speed at location $(x, y)$ during time interval $\tau$ can be estimated by the corresponding average vehicle density, given as follows:

$$
V_{\tau}(x, y)=V_{x, y}\left(\rho_{\tau}(x, y)\right), \forall(x, y) \in \Omega, \tau \in \Lambda .
$$

The local travel time per unit distance of travel incurred by all vehicles at location $(x, y)$ during time interval $\tau$ can be estimated as follows:

$$
t_{\tau}(x, y)=\frac{1}{V_{\tau}(x, y)}, \forall(x, y) \in \Omega, \tau \in \Lambda .
$$

Following Wong et al. (2008), we define the fare of a taxi ride to be the monetary cost that is charged to a customer. The taxi fare consists of a mileage charge and a delay-based charge, and can be formulated as follows:

$$
\varpi_{\tau}(x, y)=\left\{\begin{array}{ll}
\kappa_{1}, & \text { if } V_{\tau}(x, y) \geq \bar{V}, \\
\kappa_{1}+\frac{\kappa_{2}}{V_{\tau}(x, y)}, & \text { otherwise, }
\end{array} \forall(x, y) \in \Omega, \tau \in \Lambda,\right.
$$

where $\kappa_{1}$ and $\kappa_{2}$ are the charge per unit distance and the charge per unit time of waiting to a taxi customer, respectively. $\bar{V}$ is the critical speed below which a delay-based charge is applied to a taxi customer.

Each customer's generalized in-vehicle travel cost consists of two components: (1) the cost of in-vehicle travel time, and (2) the fare of the taxi ride. The local generalized in-vehicle travel cost per unit distance of travel incurred by customers at location $(x, y)$ during time interval $\tau$ can be formulated as the following general form:

$$
\mu_{\tau}(x, y)=\frac{\kappa}{V_{\tau}(x, y)}+\varpi_{\tau}(x, y), \forall(x, y) \in \Omega, \tau \in \Lambda,
$$

where $\kappa$ (in \$) denotes travelers' value of time. The first term on the right hand side of Eq. (7) represents the customers' travel cost associated with travel time whereas the second term on the right hand side of Eq. (7) represents the fare of a taxi ride.

Following Du et al. (2013) and Jiang et al. (2017), we formulate the local generalized travel cost per unit distance of travel incurred by the private car drivers at location $(x, y)$ during time interval $\tau$ as the following general form: 


$$
c_{\tau}(x, y)=\frac{\kappa}{V_{\tau}(x, y)}+\pi\left(\rho_{\tau}(x, y)\right), \forall(x, y) \in \Omega, \tau \in \Lambda
$$

where $\pi\left(\rho_{\tau}(x, y)\right)$ is a given function associated with the average density at location $(x, y)$ during time interval $\tau$. The two terms on the right hand side of Eq. (8), respectively, represent the cost associated with travel time and other costs that are dependent of the density, such as a preference for avoiding high-density regions or reducing the chance of having an accident due to congestion. The former term captures the major factor and the latter term captures the minor factor.

\subsubsection{The reactive dynamic user-optimal strategy of both occupied taxi and private car drivers}

We assume that occupied taxi and private car drivers select their routes to their destinations according to the traffic information of the previous interval. Each occupied taxi driver always selects his/her route to minimize his/her customer's generalized in-vehicle travel cost to the destination. Meanwhile, each private car driver always selects his/her route to minimize his/her generalized travel cost to his/her destination. The functional form of travel cost $\phi_{\tau}^{k}(x, y)$ for the $k$-th type of vehicle can be written as follows:

$$
\phi_{\tau}^{k}(x, y)=\tilde{\phi}_{\tau}^{k}+\min _{p} \int_{p} c_{\tau-1}^{k}(x, y) d s, \forall k \in K_{1} \cup K_{2},(x, y) \in \Omega, \tau \in \Lambda,
$$

where $p$ is any path from origin $(x, y) \in \Omega$ to the destination of the $k$-th type of vehicle, $\tilde{\phi}_{\tau}^{k}$ is the cost incurred by entering the destination of the $k$-th type of vehicle during time interval $\tau$, and

$$
c_{\tau}^{k}(x, y)=\left\{\begin{array}{l}
\mu_{\tau}(x, y), \text { if } k \in K_{1}, \\
c_{\tau}(x, y), \text { if } k \in K_{2} .
\end{array}\right.
$$

According to Huang et al. (2009), occupied taxi and private car drivers choose their paths to their individual designated $\mathrm{CBD}$ in a reactive dynamic user-optimal (DUO) manner if the following condition is satisfied:

$$
\mathbf{n}_{\tau}^{k}(x, y) / /-\nabla \phi_{\tau}^{k}(x, y), \forall k \in K_{1} \cup K_{2},(x, y) \in \Omega, \tau \in \Lambda,
$$

where // means that two vectors are parallel.

The reactive DUO conditions for the equilibrium flow pattern in the polycentric network can be formulated as follows:

$$
c_{\tau-1}^{k}(x, y) \mathbf{n}_{\tau}^{k}(x, y)+\nabla \phi_{\tau}^{k}(x, y)=0, \forall k \in K_{1} \cup K_{2},(x, y) \in \Omega, \tau \in \Lambda, t \in T_{\tau} .
$$

By combining Eqs. (11) and (12), we have

$$
\left|\nabla \phi_{\tau}^{k}\right|=c_{\tau-1}^{k}(x, y), \forall k \in K_{1} \cup K_{2},(x, y) \in \Omega, \tau \in \Lambda .
$$

The travel cost incurred by private car and occupied taxi drivers at the boundary of $\Omega$ are fixed and given, and hence we have the following boundary condition:

$$
\phi_{\tau}^{k}(x, y)=\bar{\phi}_{\tau}^{k}(x, y), \forall k \in K_{1} \cup K_{2},(x, y) \in \Gamma, \tau \in \Lambda,
$$


where $\bar{\phi}_{\tau}^{k}(x, y)$ represents the boundary value of $\phi_{\tau}^{k}(x, y)$ on $\Gamma$. By definition, we have

$$
\begin{aligned}
& \bar{\phi}_{\tau}^{m}(x, y)=\tilde{\phi}_{\tau}^{m}, \forall m \in M,(x, y) \in \Gamma_{c}^{m}, \tau \in \Lambda, \text { and } \\
& \bar{\phi}_{\tau}^{\underline{M}+m}(x, y)=\tilde{\phi}_{\tau}^{\underline{M}+m}, \forall m \in M,(x, y) \in \Gamma_{c}^{m}, \tau \in \Lambda .
\end{aligned}
$$

In summary, the travel costs incurred by occupied taxi and private car drivers can be obtained by the following two dimensional $\mathrm{HJ}$ equation:

$$
\left\{\begin{array}{l}
\left|\nabla \phi_{\tau}^{k}\right|=c_{\tau-1}^{k}(x, y), \forall k \in K_{1} \cup K_{2},(x, y) \in \Omega, \tau \in \Lambda, \\
\phi_{\tau}^{k}(x, y)=\bar{\phi}_{\tau}^{k}(x, y), \forall k \in K_{1} \cup K_{2},(x, y) \in \Gamma, \tau \in \Lambda
\end{array}\right.
$$

\subsection{The path choice strategy of vacant taxi drivers}

The opportunity customer-search models (e.g., Wong et al., 2014a, 2014b, 2015a, 2015b) can capture the fact that vacant taxi drivers can meet a customer on the way to their individual destination zone, and can be used to estimate the expected occupied travel time, the expected customer search time, cumulative profit, and the EROR of being hired. In this subsection, we develop an intervening opportunity modeling approach to modeling taxi customer-search behavior in a cell-based network. Unlike Wong et al. (2014a, 2014b, 2015a, 2015b), we assume that each taxi driver travels towards an adjacent and connected cell to maximize his/her EROR of being hired. The proposed cell-based opportunity customer-search model can still capture the fact that vacant taxi drivers can meet a customer on the way to their own destination zone, and is used to estimate the EROR of vacant taxi drivers from local customer-search. According to the value and distribution of the EROR, we then propose a mixed strategy to determine the customer-search direction.

\subsubsection{The probability of success}

The probability of success is the probability of a vacant taxi driver in a cell who can successfully pick up a customer in that cell. It is defined as the passenger demand generated from a cell over the availability of vacant taxis in that cell, subject to the condition that the probability is between zero and one inclusively. This can be expressed mathematically as follows (Wong et al., 2014a):

$$
s_{\tau}^{z}=\min \left[\frac{O_{\tau}^{z}}{A_{\tau}^{z}}, 1\right], \forall z \in Z, \tau \in \Lambda,
$$

where $s_{\tau}^{z}$ is the probability of a vacant taxi driver in cell $z$ who can successfully pick up a customer during interval $\tau, O_{\tau}^{z}$ denotes the number of recorded occupied trips starting in cell z during interval $\tau$, and $A_{\tau}^{z}$ denotes the number of recorded vacant taxis found in cell $z$ during interval $\tau$. This probability can capture the variation of the probability of successfully meeting a customer in different cells (Wong et al., 2014a). By definition, we have 


$$
\begin{aligned}
& O_{\tau}^{z}=\sum_{m \in M} \int_{\Omega_{z}}\left[\tilde{\rho}^{m}(x, y,(\tau-1) \delta)+\int_{(\tau-1) \delta}^{\tau \delta} \tilde{q}^{m}(x, y, t) d t\right] d x d y, \forall z \in Z, \tau \in \Lambda, \text { and } \\
& A_{\tau}^{z}=\int_{\Omega_{z}} \rho^{0}(x, y,(\tau-1) \delta) d x d y+\int_{(\tau-1) \delta}^{\tau \delta} \oint_{\Gamma_{z}}\left(\mathbf{f}^{0} \cdot \mathbf{n}_{z}\right)(x, y, t) d s d t, \forall z \in Z, \tau \in \Lambda,
\end{aligned}
$$

where $\mathbf{n}_{z}$ is the unit normal vector pointing towards cell $z$, and $d s$ is used to acknowledge the fact that the line integral is performed along the boundary of cell $z$.

\subsubsection{The profit and occupied travel time of a taxi ride}

The profit and occupied travel time of a vacant taxi driver who successfully picks up a customer in cell $z$ during interval $\tau$ can be formulated as follows:

$$
\begin{gathered}
P_{\tau}^{z}=\frac{\sum_{m \in M} \int_{\Omega_{z}}\left[\tilde{\rho}^{m}(x, y,(\tau-1) \delta)+\int_{(\tau-1) \delta}^{\tau \delta} \tilde{q}^{m}(x, y, t) d t\right] P_{\tau}^{m}(x, y) d x d y}{\sum_{m \in M} \int_{\Omega_{z}}\left[\tilde{\rho}^{m}(x, y,(\tau-1) \delta)+\int_{(\tau-1) \delta}^{\tau \delta} \tilde{q}^{m}(x, y, t) d t\right] d x d y}, \text { and } \\
T_{\tau}^{z}=\frac{\sum_{m \in M} \int_{\Omega_{z}}\left[\tilde{\rho}^{m}(x, y,(\tau-1) \delta)+\int_{(\tau-1) \delta}^{\tau \delta} \tilde{q}^{m}(x, y, t) d t\right] \eta_{\tau}^{m}(x, y) d x d y}{\sum_{m \in M} \int_{\Omega_{z}}\left[\tilde{\rho}^{m}(x, y,(\tau-1) \delta)+\int_{(\tau-1) \delta}^{\tau \delta} \tilde{q}^{m}(x, y, t) d t\right] d x d y},
\end{gathered}
$$

where $P_{\tau}^{m}(x, y)$ and $\eta_{\tau}^{m}(x, y)$ can be obtained by the following two dimensional $\mathrm{HJ}$ equation, respectively:

$$
\begin{aligned}
& \left\{\begin{array}{l}
\nabla P_{\tau}^{m}(x, y) \mathbf{n}_{\tau}^{m}(x, y)=\varpi_{\tau}(x, y), \forall m \in M,(x, y) \in \Omega, \tau \in \Lambda, \\
P_{\tau}^{m}(x, y)=\bar{P}^{m}(x, y), \forall m \in M,(x, y) \in \Gamma, \tau \in \Lambda,
\end{array}\right. \text { and } \\
& \left\{\begin{array}{l}
\nabla \eta_{\tau}^{m}(x, y) \mathbf{n}_{\tau}^{m}(x, y)=t_{\tau}(x, y), \forall m \in M,(x, y) \in \Omega, \tau \in \Lambda, \\
\eta_{\tau}^{m}(x, y)=\bar{\eta}^{m}(x, y), \forall m \in M,(x, y) \in \Gamma, \tau \in \Lambda,
\end{array}\right.
\end{aligned}
$$

where $\bar{P}^{m}(x, y)$ and $\bar{\eta}^{m}(x, y)$, respectively, represent the boundary values of $P_{\tau}^{m}(x, y)$ and $\eta_{\tau}^{m}(x, y)$ on $\Gamma$.

\subsubsection{The estimation of the EROR}

In the cell-based network, a vacant taxi driver can meet a customer on the way to his/her target cell, and he/she may travel several cells before picking up a customer. Following Wong et al. (2014a), we assume that each driver makes exactly one search decision in each cell and expects to travel to a number of cells before meeting a customer. Let this number of search decisions anticipated be $L$, which is equivalent to the average number of cells traveled before picking up a customer. The number $L$ is interpreted as the long-term average for an individual driver in his/her mindset based on his/her past experience. If the number is small, then a vacant taxi driver expects to meet his/her next customer in a short distance (due to high passenger demand 
during peak hours), and the expected search area covered by this driver is small. This means that the area is defined by $L$. The boundary of this area is called the customer-search boundary (Wong et al., 2014a). Fig. 2 shows customer-search boundaries for different numbers of search decisions anticipated. When $L=3$, a vacant taxi driver can search from cell $z$ and travel through cells $z^{\prime}$ and $z^{\prime \prime}$ to cell $z^{\prime \prime \prime}$. Different from the logit-based search decision used by Wong et al. (2014a), a user optimal search decision is adopted - an adjacent cell with the maximum EROR in all prepared search decisions is selected by a vacant taxi driver.

The EROR used for formulating the route choice strategy of vacant taxi drivers is also defined on the basis of $L$, and relates to the ratio of two terms: (1) the cumulated expected profit of a vacant taxi driver who successfully picks up a customer during the customer search that starts from cell $z$ during interval $\tau$ and the driver prepares to make a local search decision $l$ times in total before meeting a customer $(1 \leq l \leq L)$, and (2) the sum of expected search time for a customer and expected occupied travel time after picking up that customer. The EROR is mathematically formulated as follows:

$$
R_{\tau}^{z, l}=\frac{P_{\tau}^{z, l}}{T_{\tau}^{z, l}+\tilde{T}_{\tau}^{z, l}},
$$

where $P_{\tau}^{z, l}, T_{\tau}^{z, l}$, and $\tilde{T}_{\tau}^{z, l}$ are defined in the following paragraph.

The cumulative expected profit of a vacant taxi driver $\left(P_{\tau}^{z, l}\right)$ represents the accumulated profit of that driver if the driver is initially located in cell $z$ during interval $\tau$ and prepares to make a local search decision $l$ times in total before meeting a customer. The cumulative expected profit of a vacant taxi driver equals the sum of two terms: the expected profit of that taxi driver if the driver successfully picks up a customer in cell $z$, and the additional expected profit gained from the subsequent cells if the driver cannot successfully picks up a customer in cell $z$. This can be formulated as follows:

$$
P_{\tau}^{z, l}= \begin{cases}P_{\tau}^{z}, & \text { if } l=1, \\ P_{\tau}^{z}+\left(1-s_{\tau}^{z}\right) \sum_{z^{\prime} \in K_{z}^{l-1}} P_{\tau}^{z^{\prime}, l-1} /\left|K_{z}^{l-1}\right|, & \text { if } 1<l \leq L,\end{cases}
$$

where $K_{z}^{l-1}$ is the set of neighbor cells of cell $z$ with the maximum EROR, i.e.,

$$
K_{z}^{l-1}=\left\{z^{\prime} \mid R_{\tau}^{z^{\prime}, l-1} \geq R_{\tau}^{z^{\prime \prime}, l-1}, \forall z^{\prime \prime} \in K_{z}\right\} \text {, and }
$$

$\left|K_{z}^{l-1}\right|$ is the number of elements in $K_{z}^{l-1}$. $K_{z}$ is the set of neighbor cells of cell z. Similarly, the expected occupied travel time of a vacant taxi driver who successfully picks up a customer during the customer search that starts from cell $z$ during interval $\tau$ and prepares to make a local search decision $l$ times in total before meeting a customer, i.e., $T_{\tau}^{z, l}$, can be formulated as follows:

$$
T_{\tau}^{z, l}= \begin{cases}T_{\tau}^{z}, & \text { if } l=1, \\ T_{\tau}^{z}+\left(1-S_{\tau}^{z}\right) \sum_{z^{\prime} \in K_{z}^{l-1}} T_{\tau}^{z^{\prime}, l-1} /\left|K_{z}^{l-1}\right|, & \text { if } 1<l \leq L .\end{cases}
$$


The expected search time of a vacant taxi driver over $l$ cells before successfully picking up a customer during the customer search that starts from cell $z$ during interval $\tau$ (i.e., $\tilde{T}_{\tau}^{z, l}$ ) can be formulated as follows:

$$
\tilde{T}_{\tau}^{z, l}= \begin{cases}0, & \text { if } l=1, \\ \left(1-s_{\tau}^{z}\right)\left(t_{\tau}^{z}+\sum_{z^{\prime} \in K_{z}^{l-1}} \tilde{T}_{\tau}^{z^{\prime}, l-1} /\left|K_{z}^{l-1}\right|\right), & \text { if } 1<l \leq L,\end{cases}
$$

where $t_{\tau}^{z}$ is the average time for taxis traveling through cell $z$.

For each cell and time period, the EROR used for formulating the route choice strategy is defined when $l=$ $L$; i.e., $R_{\tau}^{z, L}=\frac{P_{\tau}^{z, L}}{T_{\tau}^{z, L}+\tilde{T}_{\tau}^{z, L}}$.

\subsubsection{A mixed vacant taxi customer-search strategy}

In the whole cell-based network, various cells may have different expected rates of return. Vacant taxis intend to move from a cell with a lower EROR to that with a higher EROR. We assume that vacant taxi drivers can have two types of customer-search strategies: (1) the vacant taxi drivers in a cell with an acceptable EROR travel to an adjacent cell with the highest EROR, and (2) the vacant taxi drivers in a cell with an unacceptable EROR aims to directly travel to a cell with an acceptable EROR as quick as possible. Based on this assumption, we define the cells with a large enough EROR as the target cells, and define the other cells as the non-target cells. The boundedly rational user optimal principle can be used to define the set of target cells:

$$
Z_{\tau}^{*}=\left\{Z \mid R_{\tau}^{z, L} \geq R_{\tau}^{\max }(1-\varepsilon)\right\}
$$

where $R_{\tau}^{\max }=\max _{z \in Z}\left\{R_{\tau}^{z, L}\right\}$, and $\varepsilon$ is the tolerance and $0 \leq \varepsilon<1$.

It is assumed that vacant taxi drivers in target cells select their neighbor cells with the maximum EROR. When there is only one cell with the largest EROR, all vacant taxis in the same cell travel towards the same search direction. When there is more than one cell with the same maximum EROR, the vacant taxis in cell $z$ randomly travel to one of these adjacent cells $z^{L} \in K_{z}^{L}$ as their subsequent cell.

In general, the flow direction of vacant taxis in target cells satisfies the following:

$$
\mathbf{n}_{\tau}^{0}(x, y) / / \sum_{z^{\prime} \in Z_{z}^{L}} \mathbf{n}_{z z^{\prime}}, \forall(x, y) \in \bigcup_{z \in Z_{\tau}^{*}} \Omega_{z}, \tau \in \Lambda
$$

where $\mathbf{n}_{z z^{\prime}}$ is the direction from the center point of cell $z^{\prime}$ to the center point of cell $z$.

Vacant taxi drivers in a non-target cell select their own travel time-based shortest path to their closest target cell. The minimum travel time from any non-target cell to the target cell can be obtained by the following two-dimensional $\mathrm{HJ}$ equation: 


$$
\left\{\begin{array}{l}
\left|\nabla \eta_{\tau}(x, y)\right|=t_{\tau}(x, y), \forall(x, y) \in \bigcup_{z \in Z \backslash Z_{\tau}^{*}} \Omega_{z}, \tau \in \Lambda, \\
\eta_{\tau}(x, y)=0, \forall(x, y) \in \bigcup_{z \in Z_{\tau}^{*}} \Omega_{z}, \tau \in \Lambda, \\
\eta_{\tau}(x, y)=\eta_{\max }, \forall(x, y) \in \Gamma, \tau \in \Lambda,
\end{array}\right.
$$

where $\eta_{\max }$ is a very large positive constant. The first equation in Eq. (29) is the Eikonal equation which represents that vacant taxis require $t_{\tau}(x, y)$ time units to travel unit distance at location $(x, y)$ during time interval $\tau$. The second equation in Eq. (29) represents that the minimum travel time of each vacant taxi driver in a target cell to another target cell is zero; the third equation in Eq. (29) represents that the minimum travel time of each vacant taxi driver at the boundary of modeling region is very large, and hence the vacant taxis cannot travel through the CBDs and the obstacles and cannot leave the region. Vacant taxi drivers in non-target cells choose their paths to their own target cell in a dynamic reactive user-optimal manner if the following condition is satisfied:

$$
\mathbf{n}_{\tau}^{0}(x, y) / /-\nabla \eta_{\tau}(x, y), \forall(x, y) \in \bigcup_{z \in Z \backslash Z_{\tau}{ }^{2}} \Omega_{z}, \tau \in \Lambda .
$$

\subsection{Flow conservation laws}

\subsubsection{Traffic flow determination}

The following equation can be used to express the relationship between the speed, flow, and density of each type of vehicle:

$$
\mathbf{f}^{k}(x, y, t)=\rho^{k}(x, y, t) \mathbf{v}^{k}(x, y, t), \forall k \in K,(x, y) \in \Omega, t \in T .
$$

According to Eq. (31), we have $\mathbf{f}^{k}(x, y, t) / / \mathbf{v}^{k}(x, y, t)$. By definition, we have $\mathbf{v}^{k}(x, y, t) / / \mathbf{n}_{\tau}^{k}(x, y, t)$ for all $t \in T_{\tau}$, and hence we have

$$
\frac{\mathbf{v}^{k}(x, y, t)}{\left|\mathbf{v}^{k}(x, y, t)\right|}=\mathbf{n}^{k}(x, y, t), \forall k \in K,(x, y) \in \Omega, \tau \in \Lambda, t \in T_{\tau} .
$$

Substituting $|\mathbf{v}(x, y, t)|=V(x, y, t)$ into Eq. (32) and rearranging the resultant equation, we have

$$
\mathbf{v}^{k}(x, y, t)=V(x, y, t) \mathbf{n}^{k}(x, y, t), \forall k \in K,(x, y) \in \Omega, \tau \in \Lambda, t \in T_{\tau} .
$$

Substituting Eq. (33) into Eq. (31), we have

$$
\mathbf{f}^{k}(x, y, t)=\rho^{k}(x, y, t) V(x, y, t) \mathbf{n}^{k}(x, y, t), \forall k \in K,(x, y) \in \Omega, t \in T .
$$

Once an occupied taxi arrives at a CBD, the customer pays the driver and gets out of it using $\Delta t_{1}$ time units, and then it becomes a vacant taxi and leaves the boundary of the CBD with an opposite direction, where $\Delta t_{1}$ is the average time for a customer to pay the driver and get off a taxi. Hence, we have

$$
\mathbf{f}^{0}\left(x, y, t+\Delta t_{1}\right)=-\mathbf{f}^{m}(x, y, t), \forall m \in M,(x, y) \in \Gamma_{c}^{m}, t \in T .
$$




\subsubsection{The flow conservation laws of private cars and occupied taxis}

According to the flow conservation law (CL) of continuous medium mechanics, the traffic density and flow vector for each type of private car flow must satisfy the following continuity equation:

$$
\rho_{t}^{k}(x, y, t)+\nabla \cdot \mathbf{f}^{k}(x, y, t)=q^{k}(x, y, t), \forall k \in K_{1} \cup K_{2},(x, y) \in \Omega, t \in T,
$$

where $\rho_{t}^{k}(x, y, t)=\frac{\partial \rho^{k}(x, y, t)}{\partial t}, \nabla \cdot \mathbf{f}^{k}(x, y, t)=\frac{\partial f^{k}(x, y, t)}{\partial x}+\frac{\partial g^{k}(x, y, t)}{\partial y}, q^{k}(x, y, t)$ is the source term in the flow CL, which represents the number of vacant taxis per kilometers picking up customers to the $k$-th CBD at location $(x, y)$ at time $t-\Delta t_{2}$ for $k \in K_{1}$, and further represents the private car demand at location $(x, y)$ at time $t$ for $k \in K_{2}$. By definition, we have $q^{k}(x, y, t)=\bar{q}^{k-\underline{M}}(x, y, t)$ for $k \in K_{2}$, where $k-\underline{M}$ is the CBD (destination) for the $k$-th type of private car.

We assume that no vehicle is allowed to enter the obstruction through the boundary $\Gamma_{o}^{n}$ or leave the city through $\Gamma^{0}$. This implies the following boundary condition:

$$
\rho^{k}(x, y, t)=0, \forall k \in K_{1} \cup K_{2},(x, y) \in \Gamma, t \in T .
$$

Let $t=0$ and $\bar{\rho}^{k}(x, y)$, respectively, be the start time and the density of private car flow at location $(x$, $y$ ) at the beginning of the modeling period. We have the following initial condition:

$$
\rho^{k}(x, y, 0)=\bar{\rho}^{k}(x, y), \forall k \in K_{1} \cup K_{2},(x, y) \in \Gamma .
$$

The CL part of the traffic flow model for occupied taxis and private cars can be summarized as follows:

$$
\left\{\begin{array}{l}
\rho_{t}^{k}(x, y, t)+\nabla \cdot \mathbf{f}^{k}(x, y, t)=q^{k}(x, y, t), \forall k \in K_{1} \cup K_{2},(x, y) \in \Omega, t \in T, \\
\mathbf{f}^{k}(x, y, t)=-\rho^{k}(x, y, t) V(x, y, t) \mathbf{n}_{\tau}^{k}(x, y), \forall k \in K_{1} \cup K_{2},(x, y) \in \Omega, \tau \in \Lambda, t \in T_{\tau}, \\
\rho^{k}(x, y, t)=0, \forall k \in K_{1} \cup K_{2},(x, y) \in \Gamma, t \in T, \\
\rho^{k}(x, y, 0)=\bar{\rho}^{k}(x, y), \forall k \in K_{1} \cup K_{2},(x, y) \in \Omega,
\end{array}\right.
$$

where the flow direction $\mathbf{n}_{\tau}^{k}(x, y)$ and the source term $q^{k}(x, y, t)$ are the inputs of Eq. (39).

\subsubsection{The flow conservation laws of vacant taxis and customers}

We assume that customers are immediately picked up by vacant taxis when they meet, and that the vacant taxis become occupied taxis $\Delta t_{2}$ time units later, where $\Delta t_{2}$ is the average time for a vacant taxi picking up a customer after they meet. Under this assumption, either the density of vacant taxis or the density of customers at a given location is zero. This implies that we should consider three cases: (i) the density of vacant taxis is positive and the total density of customers is zero at location $(x, y)$, (ii) the density of vacant taxis is zero and the total density of customers is positive at location $(x, y)$, and (iii) the density of both vacant taxis and customers are the same and equal to zero at location $(x, y)$. For the ease of analysis, Case (iii) is divided into two sub-cases: (iii.i) $\quad-\nabla \cdot \mathbf{f}^{0}(x, y, t) \geq \sum_{m \in M} q^{m}(x, y, t) \quad, \quad$ and $\quad$ (iii.ii) 
$-\nabla \cdot \mathbf{f}^{0}(x, y, t)<\sum_{m \in M} q^{m}(x, y, t)$, where $-\nabla \cdot \mathbf{f}^{0}(x, y, t)$ is the arrival rate of vacant taxis at location $(x, y)$ at time $t$, and $\sum_{m \in M} q^{m}(x, y, t)$ is the sum of customer demand at location $(x, y)$ at time $t$.

According to the first two cases, we define the following two sets of time instants:

$$
\begin{aligned}
& T_{1}(x, y)=\left\{t: \rho^{0}(x, y, t)>0, \sum_{m \in M} \tilde{\rho}^{m}(x, y, t)=0\right\}, \text { and } \\
& T_{2}(x, y)=\left\{t: \rho^{0}(x, y, t)=0, \sum_{m \in M} \tilde{\rho}^{m}(x, y, t)>0\right\} .
\end{aligned}
$$

According to the two sub-cases of case (iii), we define the following two sets of time instants:

$$
\begin{aligned}
& T_{3}(x, y)=\left\{t: \rho^{0}(x, y, t)=\sum_{m \in M} \tilde{\rho}^{m}(x, y, t)=0,-\nabla \cdot \mathbf{f}^{0}(x, y, t) \geq \sum_{m \in M} q^{m}(x, y, t)\right\}, \text { and } \\
& T_{4}(x, y)=\left\{t: \rho^{0}(x, y, t)=\sum_{m \in M} \tilde{\rho}^{m}(x, y, t)=0,-\nabla \cdot \mathbf{f}^{0}(x, y, t)<\sum_{m \in M} q^{m}(x, y, t)\right\} .
\end{aligned}
$$

For cases (i) and (iii.i), the customer densities are zero. Hence, the densities of the vacant taxis and customers follow the following CL:

$$
\left\{\begin{array}{l}
\rho_{t}^{0}(x, y, t)+\nabla \cdot \mathbf{f}^{0}(x, y, t)=-\sum_{m \in M} \tilde{q}^{m}(x, y, t), \forall(x, y) \in \Omega, t \in T_{1}(x, y) \cup T_{3}(x, y), \\
\tilde{\rho}_{t}^{m}(x, y, t)=0, \forall m \in M,(x, y) \in \Omega, t \in T_{1}(x, y) \cup T_{3}(x, y)
\end{array}\right.
$$

A vacant taxi meeting a customer at location $(x, y)$ becomes an occupied taxi $\Delta t_{2}$ time units later. Hence, we can obtain the demand of occupied taxis at location $(x, y)$ for cases (i) and (iii.i) as follows:

$$
q^{m}\left(x, y, t+\Delta t_{2}\right)=\tilde{q}^{m}(x, y, t), \forall m \in M,(x, y) \in \Omega, t \in T_{1}(x, y) \cup T_{3}(x, y) .
$$

For cases (ii) and (iii.ii), the vacant taxi densities are zero. Hence, the densities of the vacant taxis and customers follow the following CL:

$$
\left\{\begin{array}{l}
\rho_{t}^{0}(x, y, t)=0, \forall(x, y) \in \Omega, t \in T_{2}(x, y) \cup T_{4}(x, y), \\
\tilde{\rho}_{t}^{m}(x, y, t)-\vartheta^{m}(x, y, t) \nabla \cdot \mathbf{f}^{0}(x, y, t)=\tilde{q}^{m}(x, y, t), \forall m \in M,(x, y) \in \Omega, t \in T_{2}(x, y) \cup T_{4}(x, y),
\end{array}\right.
$$

where $\vartheta^{m}(x, y, t)$ is the proportion of customers to the $m$-th CBD waiting and is defined as

$$
\vartheta^{m}(x, y, t)=\frac{\tilde{\rho}^{m}(x, y, t)}{\sum_{m^{\prime} \in M} \tilde{\rho}^{m^{\prime}}(x, y, t)}, \forall(x, y) \in \Omega, t \in T_{2}(x, y) \cup T_{4}(x, y) .
$$

Similar to Eq. (45), we can obtain the occupied taxi demand at location $(x, y)$ for cases (ii) and (iii.ii) as follows:

$$
q^{k}\left(x, y, t+\Delta t_{2}\right)=\vartheta^{k}(x, y, t) \nabla \cdot \mathbf{f}^{0}(x, y, t), \forall k \in K_{1},(x, y) \in \Omega, t \in T_{2}(x, y) \cup T_{4}(x, y) .
$$

Note that type $k$-th occupied taxis head to the $k$-th CBD as their destination. 


\section{Solution algorithm}

In this paper, we use the Lax-Friedrichs Hamiltonian to solve the proposed HJ equations based on a rectangular mesh. The Lax-Friedrichs sweeping scheme is extremely simple to carry out, no matter whether the Hamiltonian is convex or not, and no matter how complicated the Hamiltonian could be (Kao et al., 2004). The solution process is mainly based on the adjacent grid information during the same time interval. In this section, we also describe the Lax-Friedrichs scheme to solve the proposed CLs.

\subsection{The Lax-Friedrichs sweeping scheme used to solve the HJ equations}

\subsubsection{The Lax-Friedrichs sweeping scheme for a general static HJ equation}

We consider the following general static two-dimensional HJ equation (Zhang et al., 2006):

$$
\left\{\begin{array}{l}
H\left(\varphi_{x}, \varphi_{y}\right)=g(x, y), \forall(x, y) \in \Theta, \\
\varphi(x, y)=\bar{\varphi}(x, y), \forall(x, y) \in \Pi,
\end{array}\right.
$$

where $\varphi(x, y)$ is a two-dimensional function, $\varphi_{x}=\frac{\partial \varphi(x, y)}{\partial x}, \varphi_{y}=\frac{\partial \varphi(x, y)}{\partial y}, g(x, y)$ is a nonnegative function, $\Theta$ is a computational domain, $\Pi$ is the boundary of the domain $\Theta$, and $\bar{\varphi}(x, y)$ is the boundary value of $\varphi(x, y)$ on $\Pi$. The Hamiltonian $H$ is a nonlinear Lipschitz continuous function.

Before computing the viscosity solution of Eq. (48), a rectangular mesh $\Theta_{h}$ is used to discretize the domain $\Theta$ and $\Pi_{h}$ is used to discretize the boundary $\Pi$. Let $\Delta x$ and $\Delta y$ be the grid lengths in the $x$-direction and the $y$-direction, respectively. Let $(i, j)$ denote a grid point in $\Theta_{h}$, i.e., $\Theta_{h}=\{(i, j): 1 \leq i \leq I, 1 \leq j \leq J\}$, where $I$ and $J$ are the numbers of grids in the $x$ - and $y$-directions, respectively. Let $\varphi_{i, j}$ be the numerical solution $\varphi(x, y)$ at grid point $(i, j), \bar{\varphi}_{i, j}$ be the boundary value $\bar{\varphi}(x, y)$ at grid point $(i, j)$, and $g_{i, j}$ be the value of $g(x, y)$ at grid point $(i, j)$.

The Lax-Friedrichs numerical Hamiltonian (Osher and Shu, 1991) is the simplest among all monotone numerical Hamiltonians. In this paper, the following first-order Lax-Friedrichs sweeping scheme for general static HJ equations is adopted to update the numerical solution at grid point (i,j) (Kao et al., 2004):

$$
\varphi_{i, j}^{\text {new }}=\left(\frac{1}{\frac{\alpha_{x}}{\Delta x}+\frac{\alpha_{y}}{\Delta y}}\right)\left[g_{i, j}-H\left(\frac{\varphi_{i+1, j}-\varphi_{i-1, j}}{2 \Delta x}, \frac{\varphi_{i, j+1}-\varphi_{i, j-1}}{2 \Delta y}\right)+\alpha_{x} \frac{\varphi_{i+1, j}+\varphi_{i-1, j}}{2 \Delta x}+\alpha_{y} \frac{\varphi_{i, j+1}+\varphi_{i, j-1}}{2 \Delta y}\right],
$$

where $\alpha^{x}$ and $\alpha^{y}$ are the viscosity constants and are respectively defined as

$$
\alpha^{x}=\max _{\substack{A \leq u \leq B \\ C \leq v \leq D}}\left|H_{1}(u, v)\right|, \alpha^{y}=\max _{\substack{A \leq u \leq B \\ C \leq v \leq D}}\left|H_{2}(u, v)\right|,
$$

$H_{1}(u, v)$ and $H_{2}(u, v)$ are the partial derivatives of $H(u, v)$ with respect to the both arguments. [A, B] is the value range for $u$, and $[C, D]$ is the value range for $v . u$ and $v$ can be approximated by the following schemes: 


$$
u=\frac{\varphi_{i+1, j}-\varphi_{i-1, j}}{2 \Delta x} \text { and } v=\frac{\varphi_{i, j+1}-\varphi_{i, j-1}}{2 \Delta y}
$$

By using Eq. (49), the solution to the HJ equation (48) can be updated by Gauss-Seidel iterations with the following four alternating direction sweepings (Zhang et al., 2006):

$$
\begin{aligned}
& \text { (1) } i=1: I, j=1: J \text {; } \\
& \text { (2) } i=I: 1, j=1: J \text {; } \\
& \text { (3) } i=I: 1, j=J: 1 \text {; } \\
& \text { (4) } i=1: I, j=J: 1 \text {. }
\end{aligned}
$$

The first-order Lax-Friedrichs sweeping method for general static HJ equation (48) can be summarized as follows:

Step 0: Initialization. According to the boundary condition $\varphi(x, y)=\bar{\varphi}(x, y), \forall(x, y) \in \Pi$, assign an exact value at each grid point of $\Pi_{h}$, i.e., $\varphi_{i, j}=\bar{\varphi}_{i, j}, \forall(i, j) \in \Pi_{h}$, initialize the values at other grid points of $\Theta_{h} \backslash \Pi_{h}$, set $\varphi_{i, j}^{\text {old }}=\varphi_{i, j}, \forall(i, j) \in \Theta_{h}$, and set the convergence tolerance $\varsigma>0$.

Step 1: Sweeping following the direction $i=1: I, j=1: J$.

Step 1.1: Initialize $i=1, j=1$.

Step 1.2: Update $\phi_{i, j}^{\text {new }}$ by Eq. (49), and then set $\varphi_{i, j}=\phi_{i, j}^{\text {new }}$.

Step 1.3: If $i=I$ and $j=J$, then go to Step 2; if $j=J$, then $i=i+1, j=1$, and go to Step 1.2; otherwise, $j=j+1$ and go to Step 1.2.

Step 2: Sweeping following the direction $i=I: 1, j=1: J$.

Step 2.1: Initialize $i=I, j=1$.

Step 2.2: Update $\phi_{i, j}^{\text {new }}$ by Eq. (49), and then set $\varphi_{i, j}=\phi_{i, j}^{\text {new }}$.

Step 2.3: If $i=1$ and $j=J$, then go to Step 3; if $j=J$, then $i=i-1, j=1$, and go to Step 2.2; otherwise, $j=j+1$ and go to Step 2.2.

Step 3: Sweeping following the direction $i=I: 1, j=J: 1$.

Step 3.1: Initialize $i=I, j=J$.

Step 3.2: Update $\phi_{i, j}^{\text {new }}$ by Eq. (49), and then set $\varphi_{i, j}=\phi_{i, j}^{\text {new }}$.

Step 3.3: If $i=1$ and $j=1$, then go to Step 4; if $j=1$, then $i=i-1, j=I$, and go to Step 3.2; otherwise, $j=j-1$ and go to Step 3.2.

Step 4: Sweeping following the direction $i=1: I, j=J: 1$.

Step 4.1: Initialize $i=1, j=J$.

Step 4.2: Update $\phi_{i, j}^{\text {new }}$ by Eq. (49), and then set $\varphi_{i, j}=\phi_{i, j}^{\text {new }}$.

Step 4.3: If $i=I$ and $j=1$, then go to Step 5; if $j=1$, then $i=i+1, j=I$, and go to Step 4.2; otherwise, $j=j-1$ and go to Step 4.2.

Step 5: Convergence checking. If $\max _{(i, j) \in \Theta_{h}}\left|\phi_{i, j}^{\text {new }}-\phi_{i, j}^{\text {old }}\right| \leq \varsigma$, then stop the algorithm; otherwise, set $\varphi_{i, j}^{\text {old }}=\varphi_{i, j}, \forall(i, j) \in \Theta_{h}$ and go to Step 1. 


\subsubsection{An application of Lax-Friedrichs scheme for solving the proposed HJ equations}

The preceding Lax-Friedrichs scheme is general and can be used to solve any static HJ equation. To solve HJ equation (15), we set $\varphi(x, y)=\phi_{\tau}^{k}(x, y), \quad H\left(\varphi_{x}, \varphi_{y}\right)=\sqrt{\varphi_{x}^{2}+\varphi_{y}^{2}}=\left|\nabla \phi_{\tau}^{k}\right|, \quad g(x, y)=c_{\tau-1}^{k}(x, y)$, $\varphi_{0}(x, y)=\bar{\phi}_{\tau}^{k}(x, y), \Theta=\Omega$, and $\Pi=\Gamma$. Hence, we have

$$
H_{1}(u, v)=\frac{u}{\sqrt{u^{2}+v^{2}}} \text { and } H_{2}(u, v)=\frac{v}{\sqrt{u^{2}+v^{2}}} .
$$

HJ equation (29) can be solved by a similar Lax-Friedrichs scheme for solving HJ equation (15).

To solve HJ equation (21), we set $\varphi(x, y)=P_{\tau}^{m}(x, y), \quad H\left(\varphi_{x}, \varphi_{y}\right)=\nabla P_{\tau}^{m}(x, y) \mathbf{n}_{\tau}^{m}(x, y)$, $g(x, y)=\varpi_{\tau}^{m}(x, y), \varphi_{0}(x, y)=\bar{P}^{m}(x, y), \Theta=\Omega$, and $\Pi=\Gamma$. Let $\zeta_{\tau}^{m}(x, y)$ be the angle between the direction of the movement of occupied taxis to the $m$-th CBD and the $x$-axis at location $(x, y)$ during interval $\tau$, and hence we have $\mathbf{n}_{\tau}^{m}(x, y)=\left(\cos \zeta_{\tau}^{m}(x, y), \sin \zeta_{\tau}^{m}(x, y)\right)$. According to (52), we have $H_{1}(u, v)=\cos \zeta_{\tau}^{m}(x, y)$ and $H_{2}(u, v)=\sin \zeta_{\tau}^{m}(x, y)$. HJ equation (22) can be solved by a similar Lax-Friedrichs scheme for solving HJ equation (21).

\subsection{The Lax-Friedrichs scheme used to solve the CLs}

\subsubsection{The Lax-Friedrichs scheme for the flow conservation laws of private cars and occupied taxis}

Before solving the proposed CLs, we solve the HJ equations to obtain the flow directions of private cars and occupied taxis. Hence, the flow directions are assumed to be known beforehand when solving the proposed CLs of private cars and occupied taxis. Let $\mathbf{n}_{i j}^{k}(t)$ be the flow direction of the $k$-th type of vehicle at grid point $(i, j)$ at time $t$, and $\zeta_{i j}^{k}(t)$ be the corresponding angle of the flow direction $\mathbf{n}_{i j}^{k}(t)$. By definition, we have $\mathbf{n}_{i j}^{k}(t)=\mathbf{n}_{\tau}^{k}\left(x_{i}, y_{i}\right), \forall t \in T_{\tau}$ and hence we have $\mathbf{n}_{i j}^{k}(t)=\left(\cos \zeta_{i j}^{k}(t), \sin \zeta_{i j}^{k}(t)\right)$, where $\left(x_{i}, y_{i}\right)$ is the center of grid point $(i, j)$. Let $\rho_{i, j}^{k}(t)$ be the numerical solution of $\rho^{k}(x, y, t)$ at grid point $(i, j)$ at time $t$. Let $\mathbf{f}_{i, j}^{k}(t)=\left(f_{i, j}^{k}(t), g_{i, j}^{k}(t)\right)$ be the flow of the $k$-th type of vehicle at grid point $(i, j)$ at time $t$, where $f_{i, j}^{k}(t)$ and $g_{i, j}^{k}(t)$ are the flows of the $k$-th type of vehicle in the $x$-and $y$-directions at grid point $(i, j)$ at time $t$, respectively. According to Eq. (34), we have

$$
\begin{aligned}
& f_{i, j}^{k}(t)=-\rho_{i, j}^{k}(t) V_{i, j}(t) \cos \zeta_{i j}^{k}(t) \text { and } \\
& g_{i, j}^{k}(t)=-\rho_{i, j}^{k}(t) V_{i, j}(t) \sin \zeta_{i j}^{k}(t),
\end{aligned}
$$

where $V_{i, j}(t)$ is the speed of all vehicle flows at grid point $(i, j)$ at time $t$. According to Eq. (1), $V_{i, j}(t)$ is a function of the vehicle density $\rho_{i, j}^{k}(t)$, and hence both $f_{i, j}^{k}(t)$ and $g_{i, j}^{k}(t)$ are functions of $\rho_{i, j}^{k}(t)$.

The following scheme can be used to update the density of private cars and occupied taxis:

$$
\rho_{i, j}^{k}(t+\Delta t)=\rho_{i, j}^{k}(t)-\frac{\Delta t}{\Delta x}\left(\hat{f}_{i+\frac{1}{2}, j}^{k}(t)-\hat{f}_{i-\frac{1}{2}, j}^{k}(t)\right)-\frac{\Delta t}{\Delta y}\left(\hat{g}_{i, j+\frac{1}{2}}^{k}(t)-\hat{g}_{i, j-\frac{1}{2}}^{k}(t)\right)+q_{i, j}^{k}(t) \Delta t,
$$


where $\Delta t$ is the time step for updating vehicle density, $q_{i, j}^{k}(t)$ is the given demand of the $k$-th type of vehicle at grid point $(i, j)$ at time $t$, and $\hat{f}_{i+\frac{1}{2}, j}^{k}(t)$ and $\hat{g}_{i, j+\frac{1}{2}}^{k}(t)$ are the numerical fluxes of the $k$-th type of vehicle in the $x$ - and $y$-directions at time $t$, respectively.

Following Du et al. (2013), we use the Lax-Friedrichs flux, which is a monotone flux:

$$
\begin{aligned}
& \hat{f}_{i+\frac{1}{2}, j}^{k}(t)=\frac{1}{2}\left[f_{i, j}^{k}(t)+f_{i+1, j}^{k}(t)-\alpha_{f}^{k}(t)\left(\rho_{i+1, j}^{k}(t)-\rho_{i, j}^{k}(t)\right)\right], \text { and } \\
& \hat{g}_{i, j+\frac{1}{2}}^{k}(t)=\frac{1}{2}\left[g_{i, j}^{k}(t)+g_{i, j+1}^{k}(t)-\alpha_{g}^{k}(t)\left(\rho_{i, j+1}^{k}(t)-\rho_{i, j}^{k}(t)\right)\right],
\end{aligned}
$$

where

$$
\begin{aligned}
& \alpha_{f}^{k}(t)=\max _{(i, j) \in \Theta_{h}}\left|\frac{\partial f_{i, j}^{k}(t)}{\partial \rho_{i, j}^{k}(t)}\right| \text { and } \\
& \alpha_{g}^{k}(t)=\max _{(i, j) \in \Theta_{h}}\left|\frac{\partial g_{i, j}^{k}(t)}{\partial \rho_{i, j}^{k}(t)}\right| .
\end{aligned}
$$

\subsubsection{The Lax-Friedrichs scheme for the flow conservation laws of vacant taxis and customers}

Let $h_{i, j}^{0}(t)$ be the number of vacant taxis arriving at grid point $(i, j)$ during $[t, t+\Delta t]$. The following scheme can be used to update $h_{i, j}^{0}(t)$ :

$$
h_{i, j}^{0}(t)=-\frac{\Delta t}{\Delta x}\left(\hat{f}_{i+\frac{1}{2}, j}^{0}(t)-\hat{f}_{i-\frac{1}{2}, j}^{0}(t)\right)-\frac{\Delta t}{\Delta y}\left(\hat{g}_{i, j+\frac{1}{2}}^{0}(t)-\hat{g}_{i, j-\frac{1}{2}}^{0}(t)\right)
$$

where $\hat{f}_{i+\frac{1}{2}, j}^{0}(t)$ and $\hat{g}_{i, j+\frac{1}{2}}^{0}(t)$ are the numerical fluxes of vacant taxis in the $x$ - and $y$-directions at time $t$, respectively. Similar Lax-Friedrichs schemes as in Eqs. (56) and (57) can be used to compute them.

The following scheme can be used to update the densities of vacant taxis and customers:

$$
\begin{aligned}
& \rho_{i, j}^{0}(t+\Delta t)=\left\{\begin{array}{l}
0, \text { if } \rho_{i, j}^{0}(t)+h_{i, j}^{0}(t)<\sum_{m \in M}\left(\tilde{\rho}_{i, j}^{m}(t)+\tilde{q}_{i, j}^{m}(t) \Delta t\right), \\
\rho_{i, j}^{0}(t)+h_{i, j}^{0}(t)-\sum_{m \in M}\left(\tilde{\rho}_{i, j}^{m}(t)+\tilde{q}_{i, j}^{m}(t) \Delta t\right), \text { otherwise, }
\end{array}\right. \text { and } \\
& \tilde{\rho}_{i, j}^{m}(t+\Delta t)=\left\{\begin{array}{l}
0, \text { if } \rho_{i, j}^{0}(t)+h_{i, j}^{0}(t) \geq \sum_{m \in M}\left(\tilde{\rho}_{i, j}^{m}(t)+\tilde{q}_{i, j}^{m}(t) \Delta t\right), \\
\tilde{\rho}_{i, j}^{m}(t)+\tilde{q}_{i, j}^{m}(t) \Delta t-\frac{\left[\rho_{i, j}^{0}(t)+h_{i, j}^{0}(t)\right]\left(\tilde{\rho}_{i, j}^{m}(t)+\tilde{q}_{i, j}^{m}(t) \Delta t\right)}{\sum_{m^{\prime} \in M}\left[\tilde{\rho}_{i, j}^{m^{\prime}}(t)+\tilde{q}_{i, j}^{m^{\prime}}(t) \Delta t\right]}, \text { otherwise, }
\end{array}\right.
\end{aligned}
$$

where $\tilde{\rho}_{i, j}^{m}(t)$ and $\tilde{q}_{i, j}^{m}(t)$ are respectively the customer density and customer demand at grid point $(i, j)$ at time $t$. Note that if vacant taxis did not pick up customers during $(t, t+\Delta t]$, then $\sum_{m \in M}\left(\tilde{\rho}_{i, j}^{m}(t)+\tilde{q}_{i, j}^{m}(t) \Delta t\right)$ would be the density of customers at grid point $(i, j)$ at $t+\Delta t$, and $\rho_{i, j}^{0}(t)+h_{i, j}^{0}(t)$ would be the density of vacant taxis at grid point $(i, j)$ at $t+\Delta t$. Because we assume that customers are immediately picked up by 
vacant taxis when they meet, either the density of vacant taxis or the density of customers at a given location is zero. Therefore, the density of vacant taxis at grid point $(i, j)$ at $t+\Delta t$ is zero if

$\rho_{i, j}^{0}(t)+h_{i, j}^{0}(t)<\sum_{m \in M}\left(\tilde{\rho}_{i, j}^{m}(t)+\tilde{q}_{i, j}^{m}(t) \Delta t\right)$; otherwise, the density of customers at grid point $(i, j)$ at $t+\Delta t$ is zero. However, we don't know beforehand that either $\rho_{i, j}^{m}(t)$ or $\left.\rho_{i, j}^{0}(t)\right)$ is zero.

\subsection{The overall procedure for solving the taxi traffic assignment problem}

The overall solution algorithm for solving the proposed DTTA model can be stated as follows:

Step 1: Initialization. Initialize the probability of success for all cells, and the densities of private cars, occupied taxis, vacant taxis, and customers. Set the number of search decisions anticipated $L>0$, the tolerance $\varepsilon>0, \Delta t>0, t=0$, and the time interval $\tau=0$.

Step 2: Determining traffic flow directions.

Step 2.1: Use the Lax-Friedrichs sweeping method to solve HJ equation (15) and obtain the flow directions of occupied taxis and private cars by Eq. (11).

Step 2.2: Substitute the obtained flow directions of occupied taxis to HJ equations (21) and (22). Then, use the Lax-Friedrichs sweeping method to solve the two HJ equations and obtain the fare of a taxi ride and the occupied travel time.

Step 2.3: Use the intervening opportunity model to determine the EROR for vacant taxis associated with each cell according to Eq. (23), and determine the set of target cells according to Eq. (27).

Step 2.4: Determine the flow directions of vacant taxis in target cells according to Eq. (28).

Step 2.5: Use the Lax-Friedrichs sweeping method to solve HJ equation (29) and obtain the flow directions of vacant taxis in non-target cells by Eq. (30).

Step 3: Updating density according to the CLs.

Step 3.1: Update traffic flows according to Eqs. (34) and (35).

Step 3.1: Use Lax-Friedrichs scheme (55) to solve CL (39), and update the densities of occupied taxis and private cars at time $t+\Delta t$.

Step 3.2: Use Lax-Friedrichs schemes (59) and (60) to solve CLs (44) and (46), and update the densities of vacant taxis and customers at time $t+\Delta t$.

Step 2.5: Update occupied taxi demands by Eqs. (45) and (47).

Step 4: Stop checking. If $t \geq T$, then stop; if $t \geq T_{\tau}, t=t+\Delta t, \tau=\tau+1$ and go to Step 2; otherwise, $t=t+\Delta t$ and go to Step 3 .

In Step 2, the proposed HJ equations were solved to obtain traffic flow directions. For the first iteration (i.e., $\tau=1$ ), beside the boundary grid points with predetermined trial values, large values were used as the initial trial values at all other grid points. After that, the solution to the HJ equations of interval $\tau$ was used 
as the initial solution to the HJ equations of interval $\tau+1$. The CL part of the proposed DTTA model was solved in Step 3 to update the density of each type of vehicle at each grid in the region.

\section{Numerical examples and case study}

To demonstrate the properties of the proposed model, the performance of the proposed algorithm, and the significance of using our proposed methodology for network performance prediction, we present three numerical examples and a case study in this section. In Section 5.1, we illustrate the intervening opportunity customer-search behavior of vacant taxi drivers captured by our methodology using a numerical example with a single $\mathrm{CBD}$, and show that the results obtained are consistent with our expectation. In Section 5.2, we give a numerical example with two CBDs to highlight more basic features of the studied problem that has not been discussed in the literature so far. In particular, this section demonstrates the temporal and spatial variations of system performance, the effects of different parameter values on average customer waiting time, the effect of traffic congestion mainly due to private cars on taxi customer-search efficiency, and the effect of customer demand to CBDs on the average travel time of private cars. In Section 5.3, we give a numerical example with five CBDs. This example illustrates the computation performance under various information update durations, which has important implications for online applications. In Section 5.4, we present a case study of Hong Kong Island to illustrate the better performance of the proposed DTTA model than the pure DTTA model, in which the private car DTA is given. This case study confirms the necessity of simultaneously modeling taxi and car movements for estimating network performance more accurately. All experiments were run on a computer with an Intel (R) Core(TM) 2 Quad Q9550 2.83GHz CPU and a 3.5GB RAM.

\subsection{Single $C B D$}

\subsubsection{Problem setting}

As shown in Fig. 3, we consider a rectangular domain that is $30 \mathrm{~km}$ long and $24 \mathrm{~km}$ wide. A single CBD is located at the center $(15 \mathrm{~km}, 12 \mathrm{~km})$ of the modeling domain. The following flow-density relationship is adopted (Du et al., 2013):

$$
V(x, y, t)=V_{f}(x, y) \exp \left(-\beta(x, y) \rho(x, y, t)^{2}\right), \forall(x, y) \in \Omega, t \in T,
$$

where $\beta(x, y)$ is a positive scalar related to the road condition, and $V_{f}(x, y)$ is the free-flow speed of vehicles at location $(x, y)$. We set $\beta(x, y)=2 \times 10^{-6} \mathrm{~km}^{4} / \mathrm{veh}^{2}$ and the free-flow speed is given as follows:

$$
V_{f}(x, y)=V_{\max }[1+\gamma d(x, y)],
$$

where $V_{\max }=56 \mathrm{~km} / \mathrm{h}$ is the maximum speed, $\gamma=4 \times 10^{-3} \mathrm{~km}^{-1}, d(x, y)$ is the distance from location $(x, y)$ to the center of the CBD. Travelers' value of time is $\kappa=90 \$ / \mathrm{h}$. The disutility function of congestion is $\pi(\rho)=9.0 \times 10^{-7} \rho^{2}$ (in $\$$ ). The mileage and delay-based charges to a taxi customer are $\kappa_{1}=3 \$ / \mathrm{km}$ and 
$\kappa_{2}=60 \$ / \mathrm{h}$, respectively (Wong et al., 2008). The critical speed for the delay-based charge to a taxi customer is $\bar{V}=12 \mathrm{~km} / \mathrm{h}$. We use uniform mesh grids with $\Delta x=\Delta y=0.2 \mathrm{~km}$. Each grid also represents one cell. The numerical boundary conditions for solving the HJ equations are summarized as follows:

- At the solid wall boundaries, i.e., the outer boundary of the city and the boundary of the obstruction, we let the normal numerical flux be 0 . In the HJ equations, the numerical boundary values of $\phi$ are obtained by extrapolation, and we set $\phi=10^{4}$ at the ghost points (i.e., the points on the boundaries).

- At the boundary of the compact CBD, we set $\phi=0$.

In this example, we have two scenarios to illustrate the intervening opportunity customer-search behavior of vacant taxi drivers:

- Scenario 1: All cells have a uniform probability of success at the initial time, which is denoted as $p$. (This can be achieved when both customer demand and initial vacant taxi distributions are uniform over the modeling region.)

- Scenario 2: Only the cells in the sub-area (from $7 \mathrm{~km}$ to $10 \mathrm{~km}$ in both directions) shown in Fig. 3 have a uniform probability of success at the initial time.

\subsubsection{Scenario 1: Uniform initial probability of success}

We set different initial probabilities of success in this scenario and different values for the number of customer-search decisions anticipated $(L)$ and solved the proposed HJ equations and the proposed cell-based opportunity customer-search model for $\tau=0$. Fig. 4 illustrates the expected occupied travel time, the expected vacant search time, the cumulative profit, and the EROR of vacant taxi drivers at location $(23 \mathrm{~km}, 6$ $\mathrm{km}$ ) under different values of $L$ and various probabilities of success. We can observe from the figure that the expected occupied travel time, the expected vacant search time, and the cumulative profit of vacant taxis increase as the value of $L$ increases. This result is consistent with the non-decreasing property of the three indices. One can also observe that the EROR has a quick drop when the value of $L$ is small, and has a very slow increase when the value of $L$ is large. This is because vacant taxi search time has been underestimated when the value of $L$ is small, and the EROR is about steady due to a slow increase in the expected occupied travel time, the expected vacant search time, and the cumulative profit when the value of $L$ is large. The results presented in Fig. 4 also show that a lower probability of success leads to lower expected occupied travel time, cumulative profit, and EROR, but more expected vacant search time. This result is consistent with our common sense.

We set a uniform probability of success of 0.1 for all cells and set $L=15$. Fig. 5 provides the expected occupied travel time, the expected vacant search time, the cumulative profit, and the EROR for the whole modeling domain. Fig. 6 shows the expected occupied travel time, the expected vacant search time, the cumulative profit, and the EROR at $y=10 \mathrm{~km}$. We can observe from the two figures that the expected 
occupied travel time, the cumulative profit, and the EROR are increasing from the CBD. This is because taxi occupied travel time and profit are mainly determined by the distance of a ride. A farther ride leads to more occupied travel time and more profit. However, the travel speed of vehicles nearby the CBD is low and the free flow speed is increasing from the CBD, and this leads to a low EROR of vacant taxi drivers nearby the $\mathrm{CBD}$ and the EROR is increasing from the CBD. On the contrary, the expected vacant search time slightly decreases from the CBD. This is also because the low vehicle speed nearby the CBD leads to the long customer-search time of vacant taxi drivers there.

\subsubsection{Scenario 2: Non-uniform initial probability of success}

In this scenario, we set a uniform probability of success of 0.1 for all cells in the sub-area indicated in Fig. 3. The probability of success outside the sub-area is zero. Fig. 7 provides the expected occupied travel time and the expected vacant search time when $L=15$. We can observe from the figure that the expected occupied travel time decreases from the sub-area while the expected vacant search time increases from the sub-area. This is because vacant taxi drivers in the sub-area have a higher probability to meet a customer and hence have less expected vacant search time and more expected occupied travel time. Fig. 8 provides the EROR of each cell when $L$ takes different values. We can observe from the figure that the EROR decreases from the sub-area outwards. Because vacant taxi drivers' local search direction follows the principle of maximizing the EROR, vacant taxi drivers outside the sub-area select local search directions towards the sub-area. When vacant taxi drivers outside the sub-area can meet a customer in the sub-area after making enough search decisions, they have a positive EROR associated with their current position. However, the farther vacant taxi drivers are from the sub-area, more search decisions are made to meet a customer in the sub-area and thus the lower is their EROR. Fig. 8 also shows that the area with a positive EROR enlarges as the value of $L$ increases, because vacant taxi drivers are expected to make more search decisions before meeting a customer in the sub-area.

\subsection{Two CBDs with a single obstruction}

\subsubsection{Problem setting}

As shown in Fig. 9, we consider a rectangular computational domain with two CBDs and one lake. The center of CBD 1 is located at $(8 \mathrm{~km}, 12 \mathrm{~km})$. The center of CBD 2 is located at $(22 \mathrm{~km}, 12 \mathrm{~km})$. The lake is located at $(15 \mathrm{~km}, 12 \mathrm{~km})$. The modeling period is $5 \mathrm{~h}$. We assume that there is no traffic at the beginning of the modeling period, and that no additional cost is incurred by entering the CBDs. The traffic demand functions for private cars and taxi customers are respectively defined as follows:

$$
\begin{aligned}
& \bar{q}^{m}(x, y, t)=\bar{q}_{\max }^{C}\left[1-\gamma_{1} d_{m}(x, y)\right] g(t), \text { and } \\
& \tilde{q}^{m}(x, y, t)=\tilde{q}_{\max }^{T C} g(t),
\end{aligned}
$$


where $\bar{q}_{\max }^{C}=120 \mathrm{veh} / \mathrm{km}^{2} / \mathrm{h}$ and $\tilde{q}_{\max }^{T C}=30$ person $/ \mathrm{km}^{2} / \mathrm{h}$ are the maximum demands of private cars and taxi customers, respectively. $\gamma_{1}=0.01 \mathrm{~km}^{-1}$, which is a positive scalar. $d_{m}(x, y)$ is the distance from location $(x, y)$ to the center of the $m$-th CBD. $g(t)$ is a non-negative and time-varying function defined as follows:

$$
g(t)=\left\{\begin{array}{lc}
t, & t \in[0,1], \\
1, & t \in[1,2], \\
-0.8(t-3)+0.2 & t \in[2,3], \\
0.2, & t \in[3,4], \\
0, & t \in[4,5]
\end{array}\right.
$$

In this example, the speed-density function (61) is also used, in which $\beta(x, y)=2 \times 10^{-6} \mathrm{~km}^{4} / \mathrm{veh}^{2}$, and the free-flow speed is given as follows:

$$
V_{f}(x, y)=V_{\max }\left[1+\gamma \min _{m}\left\{d_{m}(x, y)\right\}\right],
$$

where $V_{\max }=56 \mathrm{~km} / \mathrm{h}$ and $\gamma=4 \times 10^{-3} \mathrm{~km}^{-1}$.

The average times for a customer to get off and get on a taxi are, respectively, $\Delta t_{1}=60 \mathrm{~s}$ and $\Delta t_{2}=30 \mathrm{~s}$. The time step for updating vehicle density is $\Delta t=1 \mathrm{~s}$. Unless otherwise specified, the length of information time $\delta=120$ s (i.e., $2 \mathrm{~min}$ ), the number of search decisions anticipated $L=15$, and the tolerance $\varepsilon=0.4$.Initially, the densities of both private cars and occupied taxis are zero, the vacant taxis are uniformly distributed in the whole modeling region, the density of vacant taxis is $25 \mathrm{veh} / \mathrm{km}^{2}$, and all cells have the same probability of success. Other parameter values are the same as those in Section 5.1.

\subsubsection{The temporal and spatial variations of the system performance}

Using the proposed model, we can obtain the cumulative car demand of the whole modeling domain up to time $t(\bar{Q}(t))$ and the cumulative number of cars that enter the two CBDs up to time $t\left(\bar{F}_{C B D}(t)\right)$ as follows, respectively:

$$
\begin{aligned}
& \bar{Q}(t)=\sum_{m \in M} \int_{0}^{t} \iint_{\Omega} \bar{q}^{m}(x, y, w) d x d y d w, \text { and } \\
& \bar{F}_{C B D}(t)=\sum_{m \in M} \int_{0}^{t} \oint_{\Gamma_{c}^{m}} \mathbf{f}^{\underline{M}+m}(x, y, w) \mathbf{n}^{\underline{M}+m}(x, y, w) d s d w
\end{aligned}
$$

The cumulative customer demand of the whole modeling domain up to time $t(\tilde{Q}(t))$, and the cumulative number of customers picked up by taxis and arrived at the CBDs up to time $t\left(\tilde{F}_{\text {picked }}(t)\right.$ and $\left.\tilde{F}_{C B D}(t)\right)$ can be formulated as follows:

$$
\begin{aligned}
& \tilde{Q}(t)=\sum_{m \in M} \int_{0}^{t} \iint_{\Omega} \tilde{q}^{m}(x, y, w) d x d y d w \\
& \tilde{F}_{\text {picked }}(t)=\sum_{m \in M} \int_{0}^{t} \iint_{\Omega} q^{m}\left(x, y, w+\Delta t_{2}\right) d x d y d w, \text { and }
\end{aligned}
$$




$$
\tilde{F}_{C B D}(t)=\sum_{m \in M} \int_{0}^{t} \oint_{\Gamma_{c}^{m}} \mathbf{f}^{m}(x, y, w) \mathbf{n}^{m}(x, y, w) d s d w .
$$

By changing the value of $t$, the above cumulative values for the whole study period can be obtained.

Fig. 10(a) shows the cumulative car demand of the whole modeling domain and the cumulative number of cars that enter the two CBDs over the studied period. We can see that the two curves overlap and remain flat after $t=4.51 \mathrm{~h}$, which demonstrates that all of the private cars have entered the CBD by that time. Fig. 10(b) shows the cumulative customer demand of the whole modeling domain and the cumulative number of customers picked up by taxis and arrived at the CBDs. We can also see that the three curves overlap and remain stable at $t=4.54 \mathrm{~h}$, which demonstrates that all of the customers have been picked up by taxis and arrived at the CBD by that time.

Fig. 11 shows the average speed, the EROR, and the probability of success of the modeling region. We can observe from the figure that the average speed initially decreases due to the growth of traffic demand. Although the traffic demand starts to decrease from $t=2 \mathrm{~h}$, the average speed continues to decrease until $t=$ $2.5 \mathrm{~h}$. This is because the regions around the CBDs are still congested. As the demand of private cars decreases further, the average speed grows up. Different from the average speed, the average probability of success increases with an increasing customer demand and decreases with a decreasing customer demand, and decreases to zero at $t=4.06 \mathrm{~h}$ due to no customer demand after $t=4 \mathrm{~h}$. The average EROR increases quickly at the beginning due to the growth of average probability of success, and then drops due to the increase in network congestion (i.e., decrease in average speed). After $t=2.5 \mathrm{~h}$, as the average speed increases, the average EROR grows up again for the following $0.5 \mathrm{~h}$ and then decreases because of the decreasing customer demand. As the average probability of success drops to zero, the average EROR also drops to zero.

To illustrate the distributions of and changes in congestion of the modeling domain, we graphically show the temporal and spatial distributions of the density of private cars within the modeling region in Fig. 12. We can observe that the density of private cars is low at the beginning and the traffic is in the non-congested state (see Fig. 12(a)). As the traffic demand grows, the areas around the CBD boundaries become congested (see Figs. 12(b) and 12(c)). Although the demand of private cars starts to decrease since $t=2 \mathrm{~h}$, the areas around the CBDs are still in the congested state at $t=2.5 \mathrm{~h}$ (Fig. 12(d)). As the demand of private cars decreases further, all parts of the city return to the non-congested state (Figs. 12(e) and (f)). Finally, all of the private cars have entered the CBDs and there is no private cars in the city at $t=5 \mathrm{~h}$.

Fig. 13 shows the temporal and spatial distributions of the probability of success for taxi customer-search within the modeling region. We can observe that the areas with a high probability of success are small at the beginning due to low customer demand (see Fig. 13(a)). As the customer demand grows, the areas with a high probability of success increase (see Figs. 13(b) and (c)) until $t=2 \mathrm{~h}$ (see Fig. 11). As the customer demand decreases, the areas with a high probability of success also decrease (Figs. 13(d), (e), and (f)). Finally, all customers have arrived at the CBDs and the probability of success of each cell decreases to zero at $t=4.06 \mathrm{~h}$. 
Fig. 14 shows the temporal and spatial distributions of the EROR for taxi customer-search within the modeling region. We can observe that the EROR is low from $t=1.5 \mathrm{~h}$ to $2.5 \mathrm{~h}$ for the areas around the CBDs (see Figs. 14(b), (c), and (d)) due to high congestion in those areas (see Fig. 12(b), (c), and (d)). Before and after this period, the vacant taxi drivers have a higher EROR (see Figs. 14(a), (e), and (f)).

\subsubsection{The effect of the number of search decisions anticipated}

The number of search decisions anticipated $L$ is an important parameter in the intervening opportunity model (Wong et al. 2014a). We solved the proposed DTTA model with different values of $L$. Using the proposed model, we can obtain the average customer waiting time as follows:

$$
\tilde{W}=\frac{\sum_{m \in M} \int_{0}^{\bar{T}}\left[\tilde{Q}(t)-\tilde{F}_{\text {picked }}(t)\right] d t}{\tilde{Q}(\bar{T})}=\frac{\sum_{m \in M} \int_{0}^{\bar{T}} \int_{0}^{t} \iint_{\Omega}\left[\tilde{q}^{m}(x, y, w)-q^{m}\left(x, y, w+\Delta t_{2}\right)\right] d x d y d w d t}{\sum_{m \in M} \int_{0}^{\bar{T}} \iint_{\Omega} \tilde{q}^{m}(x, y, t) d x d y d t},
$$

where the numerator on the rightmost hand side of the above equation is the total customer waiting time over the whole modeling domain and the studied period, and the denominator is the total number of customers generated over the whole modeling domain and the studied period.

The average customer waiting time against $L$ is shown in Fig. 15. It can be seen that the average customer waiting time drops firstly and has an increasing tendency later as $L$ grows up, and that there is an optimal value of $L$. This implies that the value of $L$ has to be calibrated carefully to estimate the waiting time accurately.

\subsubsection{The effect of the tolerance}

We solved the proposed DTTA model with different values of tolerance $\varepsilon$ and graphically show the average customer waiting time against $\varepsilon$ in Fig. 16. From this figure, we can observe that the average customer waiting time drops firstly and increases later as $\varepsilon$ grows up, and the average customer waiting time has a minimum value at $\varepsilon=0.4$. An explanation for the result presented in Fig. 16 is that when $\varepsilon$ is too small, vacant taxi drivers tend to search for customers in limited areas (with a very high EROR) and get together, and this leads to low search efficiency. In contrast, when $\varepsilon$ is too large, the vacant taxi drivers mainly search for customers locally (i.e., in adjacent areas) without considering cells with a high EROR due to high customer demand.

\subsubsection{The effect of the length of information update interval}

We set different lengths of information update interval, and solved the proposed DTTA model. The average travel time of private cars and the average customer waiting time are graphically shown in Fig. 17. We can see that shortening information update interval can effectively improve traffic mobility as measured 
by the average travel time of private cars and taxi customer-search efficiency as measured by the average customer waiting time.

\subsubsection{The effect of traffic congestion}

We set different values of the maximum demand of private cars, and solved the proposed DTTA model. The average travel time of private cars and the average customer waiting time are graphically shown in Fig. 18. We can see that the average travel time of private cars increases with the demand, because the system is more congested when the demand is higher. Moreover, higher private car demand leads to a more significant increase in the search time, and hence lower taxi customer-search efficiency, due to a more congested network.

\subsubsection{The effect of customer demand}

We set different values of the maximum demand of customers, and solved the proposed DTTA model. The average travel time of private cars and the average customer waiting time are graphically shown in Fig. 19. We can see that the average travel time of private cars slightly increases when customer demand grows up. This is because higher customer demand leads to more (occupied and vacant) taxis traveling around the CBDs and more serious traffic congestion around those areas. We can also observe from the figure that the average customer waiting time increases quickly when customer demand grows up.

\subsection{Five CBDs}

As shown in Fig. 20, we consider a rectangular computational domain with five CBDs. The centers of CBDs are located at $(6 \mathrm{~km}, 5 \mathrm{~km}),(8 \mathrm{~km}, 15 \mathrm{~km}),(13 \mathrm{~km}, 10 \mathrm{~km}),(20 \mathrm{~km}, 19 \mathrm{~km})$, and $(23 \mathrm{~km}, 8 \mathrm{~km})$. The modeling period is $5 \mathrm{~h}$. The traffic demand functions for private cars and taxi customers respectively follow Eqs. (63) and (64), where $\bar{q}_{\max }^{C}=36 \mathrm{veh} / \mathrm{km}^{2} / \mathrm{h}$ and $\tilde{q}_{\max }^{T C}=12$ person $/ \mathrm{km}^{2} / \mathrm{h}$. Other parameter values are the same as those in Section 5.2.

We varied the length of information time from $0.5 \mathrm{~min}$ to $5 \mathrm{~min}$, and solved the proposed taxi traffic assignment model. The CPU times for solving the whole problem, and the total CPU times, respectively, for solving the HJ part and the CL part of the whole problem for all intervals are graphically shown in Fig. 21 . We can observe that a shorter information update interval can lead to longer CPU times for solving the whole problem and the HJ part, while the total CPU time for solving the CL part basically remains unchanged. This implies that most CPU time is spent on solving the HJ part of the DTTA model (i.e., updating the flow direction of each type of vehicle) if we shorten information update interval. Fig. 22 shows the number of intervals in the modeling period and the CPU times for solving the HJ part of the proposed model for one time interval when the length of information update interval increases. We can observe that the number of intervals decreases with the increase in the length of information update interval. However, the CPU time for solving 
the HJ part for one time interval increases with an increasing length of information update interval. This is because when the length of information update interval is small, the traffic flow state changes slightly, the initial solution of the HJ equations is closer to the optimal solution, and less CPU time is required to solve the HJ equations.

The CPU time is an important consideration for online applications. Fig. 23 plots the maximum CPU time for solving the whole problem for one interval and the ratio of the maximum CPU time to the length of information update interval against the length of information update interval. We can observe that the maximum computation time for solving the whole problem for one interval is much less than the length of information update, which implies that it satisfies the requirement for online applications.

\subsection{A case study of Hong Kong Island}

Hong Kong Island is considered in this case study. It is an island in the southern part of Hong Kong with the size of approximately $80 \mathrm{~km}^{2}$. The population and employment are heavily concentrated along its northern shore. We identify nine major districts in Hong Kong Island as shown in Fig. 24. The modeling domain is 11.4 $\mathrm{km}$ long and $14.8 \mathrm{~km}$ wide. We use uniform mesh grids with $\Delta x=\Delta y=0.2 \mathrm{~km}$.

In this case study, the survey data were collected from GPS devices previously installed in 460 urban taxis that tracked the taxi movements. By using satellite communication, the database recorded the taxis' location in terms of longitude and latitude, travel speeds, and occupational statuses at 30 seconds intervals. Hong Kong has 15,250 licensed urban taxis. The sample of 460 urban taxis represents approximately $3 \%$ of the taxi population which offer an adequate sample size to deduce the taxi movements. The GPS survey data was collected in two weeks starting from 16 to 30 August 2009 inclusively between 7:00 am and 9:30 am. We extracted the initial distribution of taxis at 7:00am and their associated occupational statuses, and the origin-destination demand of taxi customers in 15 minutes intervals based on the occupied taxi trips. An expansion factor of $2.37(=15,250 / 460 / 14)$ was applied to each observation for the total numbers of taxis and customer demand on Hong Kong Island during our concerned period.

At 7:00 am, 394 occupied taxis and 2162 vacant taxis were identified in Hong Kong Island, and 18,042 taxi customers traveled by taxis between 7:00 am and 9:30 am. Based on Travel Characteristics Survey 2011 commissioned by the Hong Kong Transport Department of The Hong Kong Special Administrative Region Government, the total demand of private cars was estimated to be 55,124 during the modeling horizon.

The traffic demand function for private cars is defined as follows:

$$
\bar{q}^{m}(x, y, t)=\bar{q}_{\max }^{C}(x, y) g(t)
$$

where $\bar{q}_{\max }^{C}(x, y)$ is the maximum demand of private cars at location $(x, y)$, and 


$$
g(t)= \begin{cases}2(t-7), & t \in[7,7.5), \\ 1, & t \in[7.5,8.5), \\ 9.5-t, & t \in[8.5,9.5) \\ 0, & \text { otherwise }\end{cases}
$$

According to the calibration by Peng (2013), the Pipes-Munjal and Drake models have the best performance in terms of fitting the MFDs for Sai Ying Pun and Causeway Bay, respectively, and the Underwood model has the best performance in terms of fitting the MFDs for other areas of Hong Kong Island. The MFDs in terms of the speed-density relationship of the Underwood, Drake, and Pipes-Munjal models are given as follows, respectively:

$$
\begin{aligned}
& V(x, y, t)=V_{f}(x, y) \exp \left(-\rho(x, y, t) / \rho_{c}(x, y)\right), \\
& V(x, y, t)=V_{f}(x, y) \exp \left(1-\sqrt{\rho(x, y, t) / \rho_{c}(x, y)}\right), \text { and } \\
& V(x, y, t)=V_{f}(x, y) \exp \left(-\frac{1}{2}\left(\rho(x, y, t) / \rho_{c}(x, y)\right)^{2}\right),
\end{aligned}
$$

where $\rho_{c}(x, y)$ is the critical density at location $(x, y)$. The two parameters, $V_{f}(x, y)$ and $\rho_{c}(x, y)$, for each area of Hong Kong Island are provided in Table 1.

The other parameter values of the proposed DTTA model are the same as those in Sections 5.2 and 5.3. The proposed solution algorithm was adopted to solve the proposed DTTA problem in Hong Kong Island. To illustrate the distributions of and changes in congestion of the studied region, we graphically show the temporal and spatial distributions of the vehicular speed within the modeling region in Fig. 25. We can observe that Sheung Wan, Central, Wan Chai, and Causeway Bay are more congested than other areas in Hong Kong Island during the peak hour from 8:00 am - 9:00 am (see Figs. 25(a), (b) and (c)), and the studied area becomes non-congested at 9:30 am (see Fig. 25(d)). These results are consistent to our observation of the temporal and spatial distributions of congestion in Hong Kong Island.

In the proposed DTTA model, private car and taxi movements are simultaneously modeled. To illustrate that it is important to model them simultaneously, we compared the results obtained by the proposed model and a pure DTTA model, in which the private car DTA is given, and real-time information from the private car DTA is used to describe the spatiotemporal characteristics of congestion. To have a fair comparison, we first determined the time-varying flow pattern of private cars using a private car DTA model without taxis, which is a special case of the proposed model, and then obtained the temporal and spatial distributions of speed of the whole network from the flow pattern. Afterwards, we solved the pure DTTA model, which is also a special case of our proposed model. The pure DTTA model let taxi drivers select their routes according to the spatiotemporal characteristics of congestion obtained from the private car DTA model without taxis, and the private car drivers directly follow their route choices determined by the private car DTA model without taxis. The performance of the two DTTA models is provided in Table 2. We can observe that, compared with using 
the pure DTTA model, using proposed DTTA model can significantly improve the network performance in terms of reducing average travel time of private cars, and average customer waiting time, average customer in-vehicle travel time, and average customer total travel time (i.e., sum of the average customer in-vehicle and waiting times). This result implies that modeling of car and taxi movements should be taken part together.

\section{Conclusions}

We develop a two-level continuum transportation system approach to modeling a DTTA problem. In the proposed model, traffic density in the system is governed by the conservation law (CL), and the flow direction is determined by the following path-choice strategies: Each private car driver chooses the path that minimizes his/her own generalized travel cost; each occupied taxi driver chooses the path that minimizes his/her customer's generalized in-vehicle travel cost, and each vacant taxi driver has a mixed strategy to determine his/her customer-search direction according to the expected rate of return (EROR). An intervening opportunity model is proposed to estimate the EROR of vacant taxi drivers during their customer-search. HJ equations are developed to capture the path choice of different drivers. Both the proposed CL and HJ equations were solved by the Lax-Friedrichs scheme, which forms the backbone of the developed algorithm. Numerical examples and a case study are presented to demonstrate the properties of the model, the computation performance of the solution algorithm, and the significance of using our methodology for estimating network performance more accurately. The results show that a more congested system leads to a higher customer search time. The results also show that shortening information update interval can effectively improve traffic mobility as measured by the average travel time of private cars and taxi customer-search efficiency as measured by the average customer waiting time, but lead to a longer CPU time for solving the whole problem.

\section{Acknowledgements}

This research was jointly supported by a grant from the Research Grants Council of the Hong Kong Special Administrative Region, China (HKU 716913E), two grants (201411159063 and 201511159095) from the University Research Committee of the University of Hong Kong, the National Natural Science Foundation of China (71431003, 71522001), and the Fundamental Research Funds for the Central Universities (JZ2016HGPB0736). We would like to express our sincere thanks to Concord Pacific Satellite Technologies Limited and Motion Power Media Limited for their kind provision of the GPS data from the taxis used for completing this study. We would also like to thank Transport Department of Hong Kong Transport Department of the Hong Kong Special Administrative Region Government to share the Travel Characteristics Survey data to us. We are grateful to the two reviewers for their constructive comments. 


\section{References}

Arnott, R., 1996. Taxi travel should be subsidized. Journal of Urban Economics 40(3), 316-333.

Ban, X., Liu, H.X., Ferris, M.C., Ran, B., 2008. A link-node complementarity model and solution algorithm for dynamic user equilibria with exact flow propagations. Transportation Research Part B 42(9), 823-842.

Bar-Gera, H., 2002. Origin-based algorithm for the traffic assignment problem. Transportation Science 36(4), 398-417.

Beckmann, M., McGuire, C.B., Winsten, C.B., 1956. Studies in the Economics of Transportation. Yale University Press, Connecticut.

Cairns, R.D., Liston-Heyes, C., 1996. Competition and regulation in the taxi industry. Journal of Public Economics 59(1), 1-15.

Carey, M., 1987. Optimal time-varying flows on congested networks. Operations Research 35(1), 58-69.

Carey, M., Subrahmanian, E., 2000. An approach to modeling time-varying flows on congested networks. Transportation Research Part B 34(3), 157-183.

Conway, A., Kamga, C., Yazici, A., Singhal, A., 2012. Challenges in managing centralized taxi dispatching at high-volume airports: Case study of John F. Kennedy International Airport. Transportation Research Record 2300, 83-90.

da Costa, D.C.T., de Neufville, R., 2012. Designing efficient taxi pickup operations at airports. Transportation Research Record 2300, 91-99.

Daganzo, C.F., Sheffi, Y., 1977. On stochastic models of traffic assignment. Transportation Science, 11(3), 253-274.

Daganzo, C.F., Geroliminis, N., 2008. An analytical approximation for the macroscopic fundamental diagram of urban traffic. Transportation Research Part B 42(9), 771-781.

De Vany, A.S., 1975. Capacity utilization under alternative regulatory constraints: An analysis of taxi markets. The Journal of Political Economy 83(1), 83-94.

Du, B., Wang, D.Z.W., 2014. Continuum modeling of park-and-ride services considering travel time reliability and heterogeneous commuters-A linear complementarity system approach. Transportation Research Part E 71, 58-81.

Du, J., Wong, S.C., Shu, C.W., Zhang, M., 2015. Reformulating the Hoogendoorn-Bovy predictive dynamic user-optimal model in continuum space with anisotropic condition. Transportation Research Part B 79, 189-217.

Du, J., Wong, S.C., Shu, C.W., Xiong, T., Zhang, M., Choi, K., 2013. Revisiting Jiang's dynamic continuum model for urban cities. Transportation Research Part B 56, 96-119.

Fernández, J.E., De Cea, J., Briones, J., 2006. A diagrammatic analysis of the market for cruising taxis. Transportation Research Part E 42(6), 498-526.

Friesz, T.L., Bernstein, D., Smith, T.E., Tobin, R.L., Wie, B., 1993. A variational inequality formulation of the dynamic networks user equilibrium problem. Operations Research 41(1), 179-191.

Friesz, T.L., Luque, J., Tobin, R.L., Wie, B.W., 1989. Dynamic network traffic assignment considered as a continuous-time optimal-control problem. Operations Research 37(6), 893-901. 
Geroliminis, N., Daganzo, C.F., 2008. Existence of urban-scale macroscopic fundamental diagrams: Some experimental findings. Transportation Research Part B 42(9), 759-770.

Geroliminis, N., Sun, J., 2011. Properties of a well-defined macroscopic fundamental diagram for urban traffic. Transportation Research Part B 45(3), 605-617.

Han, K., Szeto, W.Y., Friesz, T.L., 2015. Formulation, existence, and computation of boundedly rational dynamic user equilibrium with fixed or endogenous user tolerance. Transportation Research Part B 79, 16-49.

Han, S., 2003. Dynamic traffic modelling and dynamic stochastic user equilibrium assignment for general road networks. Transportation Research Part B 37(3), 225-249.

Ho, H.W., Wong, S.C., Sumalee, A., 2013. A congestion-pricing problem with a polycentric region and multi-class users: A continuum modeling approach. Transportmetrica A: Transport Science 9(6), 514-545.

Ho, H.W., Wong, S.C., Loo, B.P.Y., 2006. Combined distribution and assignment model for a continuum traffic equilibrium problem with multiple user classes. Transportation Research Part B 40(8), 633-650.

Hoogendoorn, S.P., Bovy, P.H.L., 2004. Dynamic user-optimal assignment in continuous time and space. Transportation Research Part B 38(7), 571-592.

Horn, M.E.T., 2002. Fleet scheduling and dispatching for demand-responsive passenger services. Transportation Research Part C 10(1), 35-63.

Hu, X., Gao, S., Chiu, Y.C., Lin, D.Y., 2012. Modeling routing behavior for vacant taxicabs in urban traffic networks. Transportation Research Record 2284, 81-88.

Huang, H.J., Lam, W.H.K., 2002. Modeling and solving the dynamic user equilibrium route and departure time choice problem in network with queues. Transportation Research Part B 36(3), 253-273.

Huang, L., Wong, S.C., Zhang, M.P., Shu, C.W., Lam, W.H.K., 2009. Revisiting Hughes' dynamic continuum model for pedestrian flow and the development of an efficient solution algorithm. Transportation Research Part B 43(1), 127-141.

Jiang, Y.Q., Xiong, T., Wong, S.C., Shu, C.W., Zhang, P., Zhang, M.P., Lam, W.H.K., 2009. A reactive dynamic continuum user equilibrium model for bi-directional pedestrian flows. Acta Mathematica Scientia 29(6), 1541-1555.

Jiang, Y.Q., Wong, S.C., Ho, H.W., Zhang, P., Liu, R.X., Sumalee, A., 2011. A dynamic traffic assignment model for a continuum transportation system. Transportation Research Part B 45(2), 343-363.

Jiang, Y.Q., Wong, S.C., Zhang P., Choi, K., 2017. Dynamic continuum model with elastic demand for a polycentric urban city. Transportation Science, doi: 10.1287/trsc.2016.0680.

Jiang, Y., Szeto, W.Y., Long, J.C., and Han, K., 2016. Multi-class dynamic traffic assignment with physical queues: Intersection-movement-based formulation and paradox. Transportmetrica A, 12(10) 878-908.

Jung, J., Chow, J.Y.J., Jayakrishnan, R., Park, J.Y., 2014. Stochastic dynamic itinerary interception refueling location problem with queue delay for electric taxi charging stations. Transportation Research Part C 40, 123-142.

Kao, C.Y., Osher, S., Qian, J., 2004. Lax-Friedrichs sweeping scheme for static Hamilton-Jacobi equations. Journal of Computational Physics 196(1), 367-391.

Lee, W.C., Cheng, B.W., 2008. Incorporating e-technology to advantage in a greener taxi industry and its 
impact on driving performance and safety. Transportation Planning and Technology 31(5), 569-588.

Lim, Y., Heydecker, B., 2005. Dynamic departure time and stochastic user equilibrium assignment. Transportation Research Part B 39(2), 97-118.

Lo, H.K., Szeto, W.Y., 2002. A cell-based variational inequality formulation of the dynamic user optimal assignment problem. Transportation Research Part B 36(5), 421-443.

Long, J.C., Huang, H.J., Gao, Z.Y., Szeto, W.Y., 2013. An intersection-movement-based dynamic user optimal route choice problem. Operations Research 61(5), 1134-1147.

Long, J.C., Szeto, W.Y., Gao, Z.Y., Huang, H.J., Shi, Q., 2016. The nonlinear equation system approach to solving dynamic user optimal simultaneous route and departure time choice problems. Transportation Research Part B 83, 179-206.

Long, J.C., Szeto, W.Y., Huang, H.J., Gao, Z.Y., 2015. An intersection-movement-based stochastic dynamic user optimal route choice model for assessing network performance. Transportation Research Part B 74, 182-217.

Ma, R., Ban, X., Pang, J.S., 2014. Continuous-time dynamic system optimum for single-destination traffic networks with queue spillbacks. Transportation Research Part B 68, 98-122.

Merchant, D.K, Nemhauser, G.L., 1978a. A model and an algorithm for the dynamic traffic assignment. Transportation Science 12(3), 183-199.

Merchant, D.K, Nemhauser, G.L., 1978b. Optimality conditions for a dynamic traffic assignment model. Transportation Science 12(3), 200-207.

Miwa, T., Ishiguro, Y., Yamamoto, T., Morikawa, T., 2013. Allocation planning for probe taxi devices based on information reliability. Transportation Research Part C 34, 55-69.

Moore, A.T., Balaker, T., 2006. Do economists reach a conclusion on taxi deregulation? Economical Journal Watch 3(1), 109-132.

Nie, Y., 2011. A cell-based Merchant-Nemhauser model for the system optimum dynamic traffic assignment problem. Transportation Research Part B 45(2), 329-342.

Nie, Y., 2010. A class of bush-based algorithms for the traffic assignment problem. Transportation Research Part B 44(1), 73-89.

Osher, S., Shu, C.W., 1991. High-order essentially nonoscillatory schemes for Hamilton-Jacobi equations. SIAM Journal on Numerical Analysis 28(4), 907-922.

Peng, J.X., 2013. Macroscopic characteristics of dense road networks. Master Dissertation, The University of Hong Kong, Hong Kong.

Ran, B., Boyce, D.E., Leblanc, L.J., 1993. A new class of instantaneous dynamic user-optimal traffic assignment models. Operations Research 41(1) 192-202.

Schroeter, J.R., 1983. A model of taxi service under fare structure and fleet size regulation. The Bell Journal of Economics 14(1), 81-96.

Smith, M.J., 1993. A new dynamic traffic and the existence and calculation of dynamic user equilibria on congestion capacity-constrained road networks. Transportation Research Part B 27(1), 49-63.

Szeto, W.Y., Jiang, Y., Sumalee, A., 2011. A cell-based model for multi-class doubly stochastic dynamic traffic assignment. Computer-Aided Civil and Infrastructure Engineering 26(8), 595-611. 
Vaughan, R.J., 1987. Urban Spatial Traffic Patterns. Pion, London.

Waller, S.T., Fajardo, D., Duell, M., Dixit, V., 2013. Linear programming formulation for strategic dynamic traffic assignment. Networks and Spatial Economics 13(4), 427-443.

Wang, D.Z.W., Du, B., 2013. Reliability-based modeling of park-and-ride service on linear travel corridor. Transportation Research Record 2333, 16-26.

Wang, D.Z.W., Du, B., 2016. Continuum modelling of spatial and dynamic equilibrium in a travel corridor with heterogeneous commuters - A partial differential complementarity system approach. Transportation Research Part B 85, 1-18.

Wie, B.W., Tobin, R.L., Carey, M., 2002. The existence, uniqueness and computation of an arc-based dynamic network user equilibrium formulation. Transportation Research Part B 36(10), 897-918.

Wong, S.C., Lee, C.K., Tong, C.O., 1998. Finite element solution for the continuum traffic equilibrium problems. International Journal for Numerical Methods in Engineering 43(7), 1253-1273.

Wong, K.I., Wong, S.C., Yang, H., Wu, J.H., 2008. Modeling urban taxi services with multiple user classes and vehicle modes. Transportation Research Part B 42(10), 985-1007.

Wong, K.I., Wong, S.C., Yang, H., 2001. Modeling urban taxi services in congested road networks with elastic demand. Transportation Research Part B 35(9), 819-842.

Wong, K.I., Wong, S.C., Yang, H., Tong, C.O., 2003. The effect of perceived profitability on the level of taxi service in remote areas. Journal of the Eastern Asia Society for Transportation Studies 5, 79-94.

Wong, R.C.P., Szeto, W.Y., Wong, S.C., 2014a. A cell-based logit-opportunity taxi customer-search model. Transportation Research Part C 48, 84-96.

Wong, R.C.P., Szeto, W.Y., Wong, S.C., 2014b. Bi-level decisions of vacant taxi drivers traveling towards taxi stands in customer-search: Modeling methodology and policy implications. Transport Policy 33, 73-81.

Wong, R.C.P., Szeto, W.Y., Wong, S.C., 2015a. A two-stage approach to modeling vacant taxi movements. Transportation Research Part C 59, 147-163.

Wong, R.C.P., Szeto, W.Y., Wong, S.C., 2015b. Sequential logit approach to modeling the customer-search decisions of taxi drivers. Asian Transport Studies 3(4), 398-415.

Yagar, S., 1971. Dynamic traffic assignment by individual path minimisation and queuing. Transportation Research 5(3), 179-196.

Yang, H., Fung, C.S., Wong, K.I., Wong, S.C., 2010. Nonlinear pricing of taxi services. Transportation Research Part A 44(5), 337-348.

Yang, H., Lau, Y.W., Wong, S.C., Lo, H.K., 2000. A macroscopic taxi model for passenger demand, taxi utilization and level of services. Transportation 27(3), 317-340.

Yang, H., Wong, S.C., 2000. A continuous equilibrium model for estimating market areas of competitive facilities with elastic demand and market externalities. Transportation Science 34(2), 216-227.

Yang, H., Wong, S.C., 1998. A network model of urban taxi services. Transportation Research Part B 32(4), 235-246.

Yang, H., Wong, S.C., Wong, K.I., 2002. Demand-supply equilibrium of taxi services in a network under competition and regulation. Transportation Research Part B 36(9), 799-819.

Yang, H., Ye, M., Tang, W.H.C., Wong, S.C., 2005. Regulating taxi services in the presence of congestion 
externality. Transportation Research Part A 39(1), 17-40.

Yin, J., Wong, S.C., Sze, N.N., Ho, H.W., 2013. A continuum model for housing allocation and transportation emission problems in a polycentric city. International Journal of Sustainable Transportation 7(4), 275-298.

Zhang, Y.T., Zhao, H.K., Qian, J., 2006. High order fast sweeping methods for static Hamilton-Jacobi equations. Journal of Scientific Computing 29(1), 25-56.

Ziliaskopoulos, A.K., 2000. A linear programming model for the single destination system optimum dynamic traffic assignment problem. Transportation Science 34(1), 37-49. 
Table 1. Parameters of the MFD of each of the areas associated with the nine CBDs.

\begin{tabular}{cccc}
\hline CBD & Model type & $V_{f}(x, y)(\mathrm{km} / \mathrm{h})$ & $\rho_{c}(x, y)\left(\mathrm{veh} / \mathrm{km}^{2}\right)$ \\
\hline Sai Ying Pun & Pipes-Munjal & 31.1 & 754 \\
Sheung Wan & Underwood & 35.8 & 709 \\
Central & Underwood & 37.3 & 716 \\
Wan Chai & Underwood & 28 & 1041 \\
Causeway Bay & Drake & 29.4 & 411 \\
Happy Valley & Underwood & 43.6 & 433 \\
Tai Koo & Underwood & 49.1 & 822 \\
Shau Kei Wan & Underwood & 38.2 & 672 \\
Chai Wan & Underwood & 36.4 & 501 \\
\hline
\end{tabular}

Table 2. Comparison of the performance of two DTTA models.

\begin{tabular}{lccc}
\hline \multicolumn{1}{c}{ Performance } & $\begin{array}{c}\text { Pure DTTA model } \\
(\mathrm{min})\end{array}$ & $\begin{array}{c}\text { Proposed DTTA model } \\
(\mathrm{min})\end{array}$ & Decrease (\%) \\
\hline Average travel time of private cars & 17.34 & 15.23 & 12.15 \\
$\begin{array}{l}\text { Average customer waiting time } \\
\text { Average customer in-vehicle travel }\end{array}$ & 21.58 & 15.64 & 27.53 \\
time & 15.44 & 14.57 & 5.62 \\
Average customer total travel time & 37.02 & 30.21 & 18.39 \\
\hline
\end{tabular}

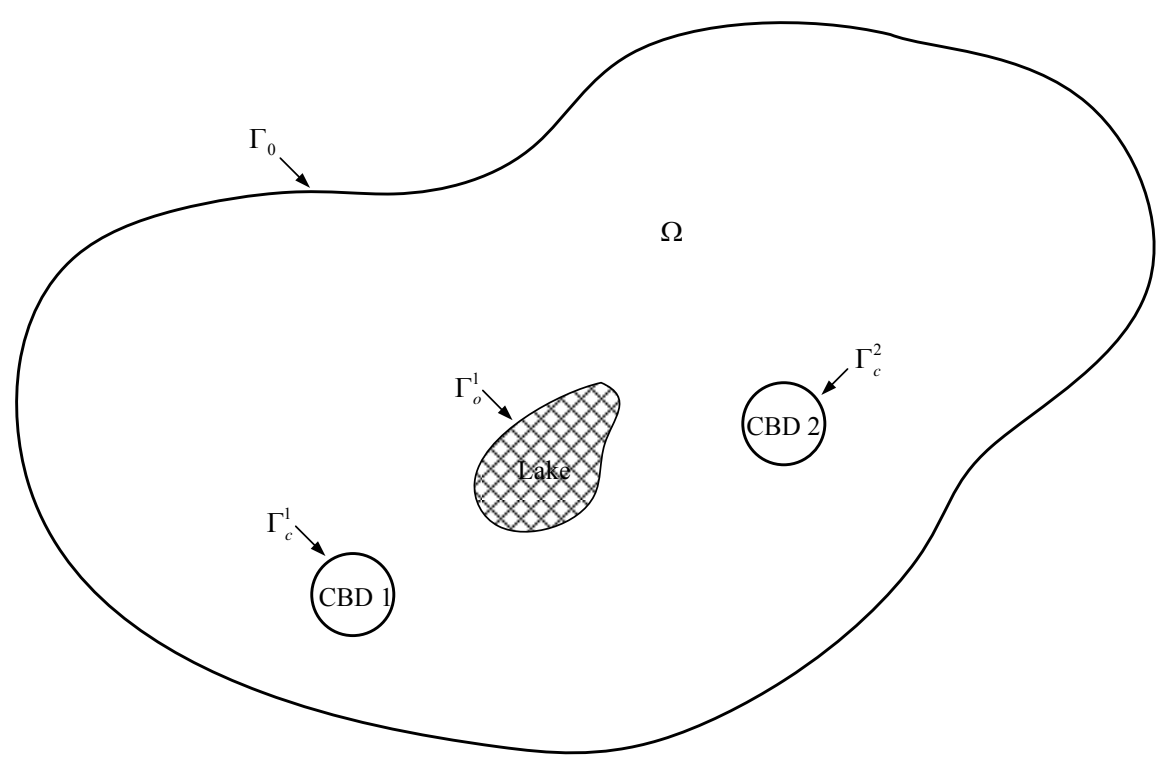

Fig. 1. The modeling domain. 


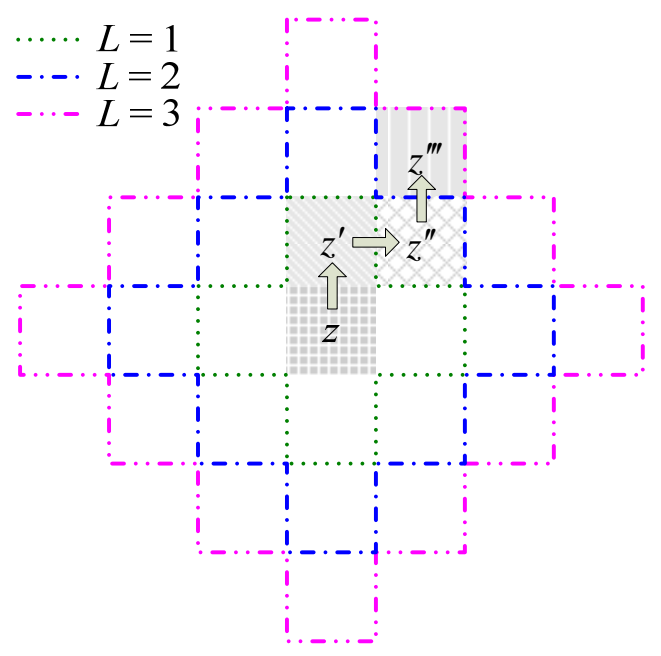

Fig. 2. Customer-search boundaries for different numbers of search decisions anticipated.

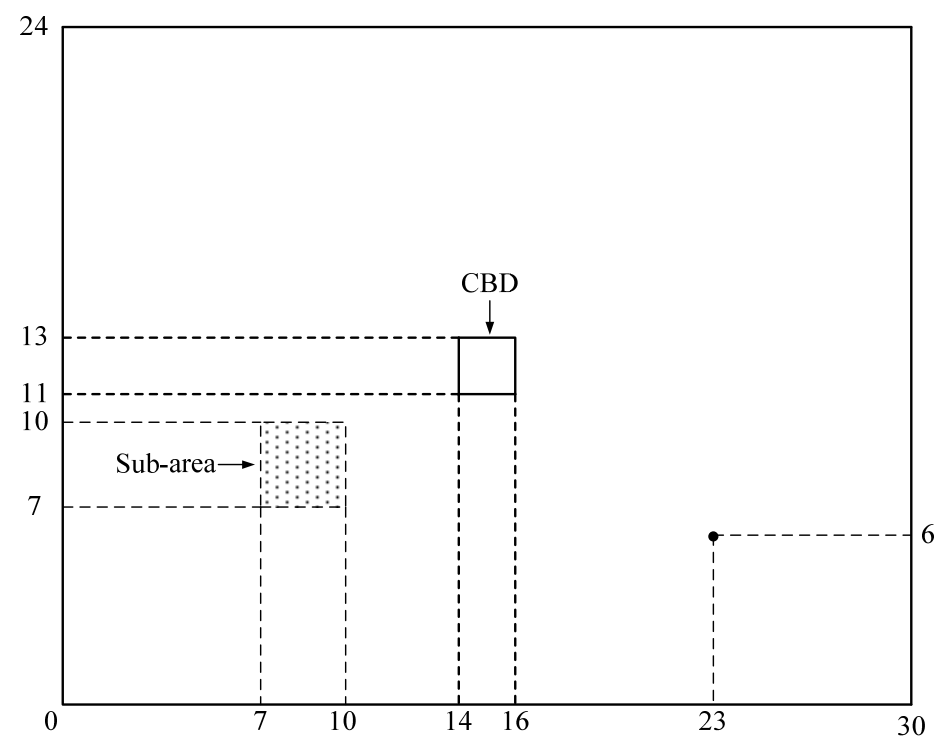

Fig. 3. The modeling domain with a single CBD. 

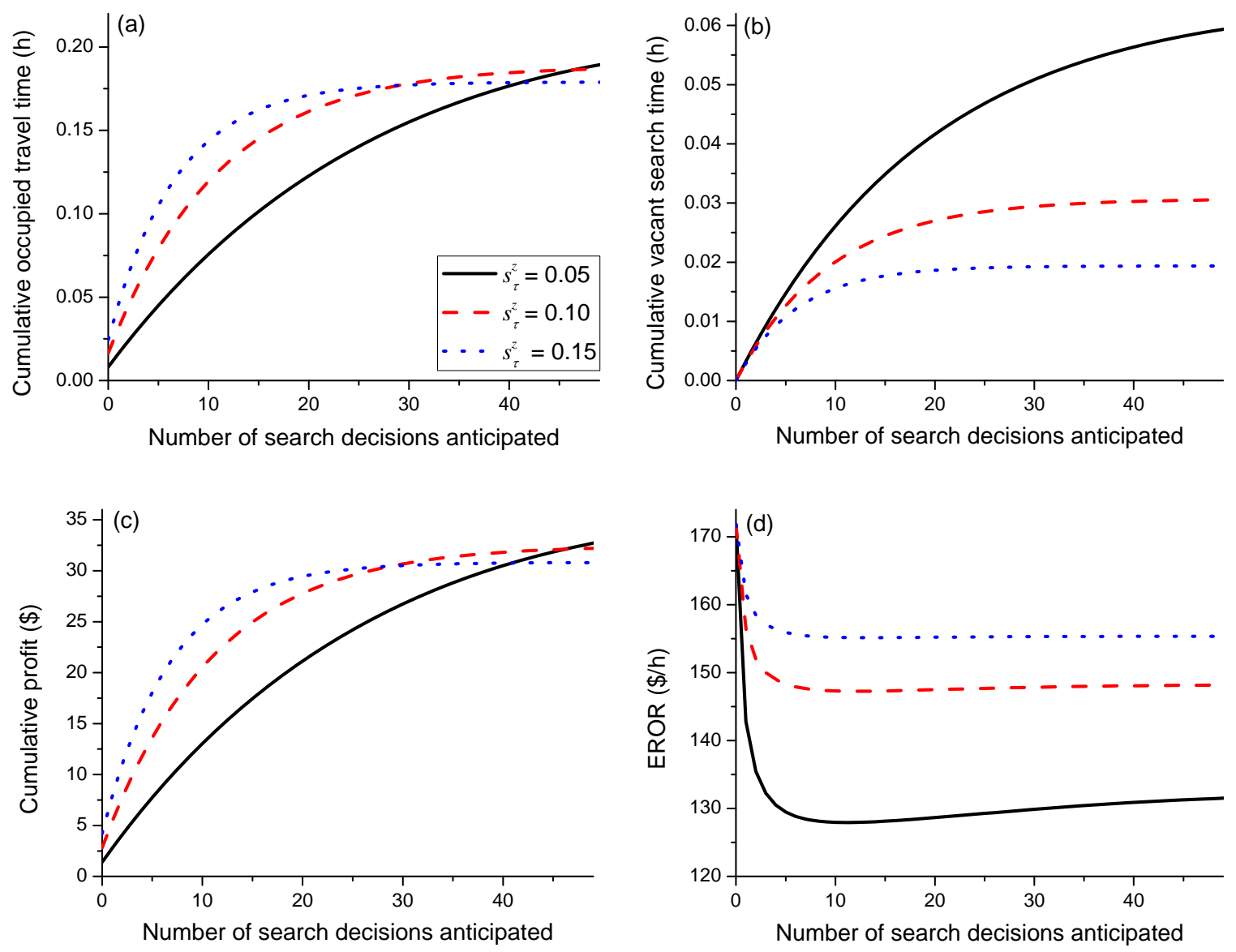

Fig. 4. The expected occupied travel time, expected vacant search time, cumulative profit, and EROR of vacant taxi drivers located at $(23 \mathrm{~km}, 6 \mathrm{~km})$ under different probabilities of success. 


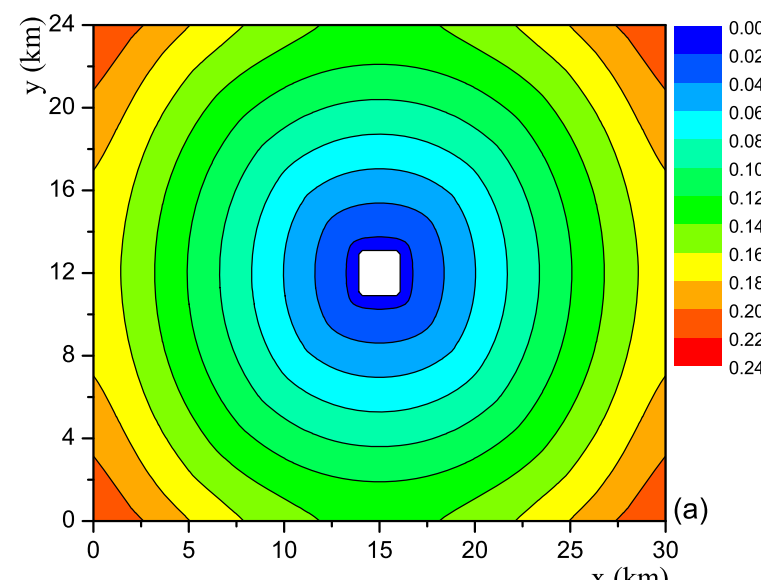

(a) Expected occupied travel time (h) $\mathrm{x}(\mathrm{km})$

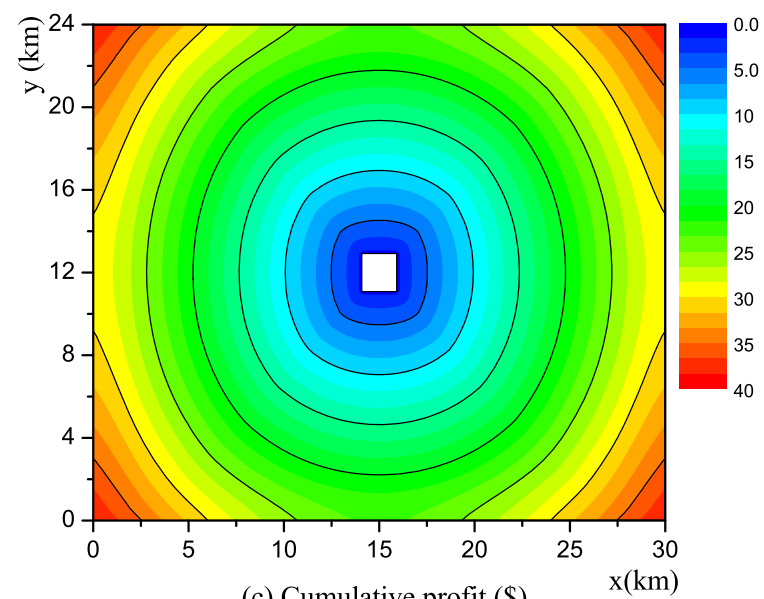

(c) Cumulative profit $(\$)$

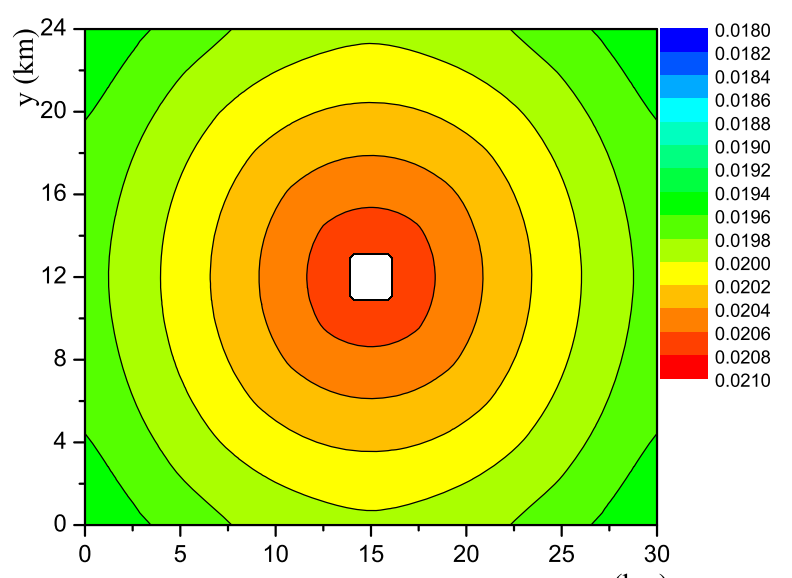

(b) Expected vacant search time (h) $\mathrm{x}(\mathrm{km})$

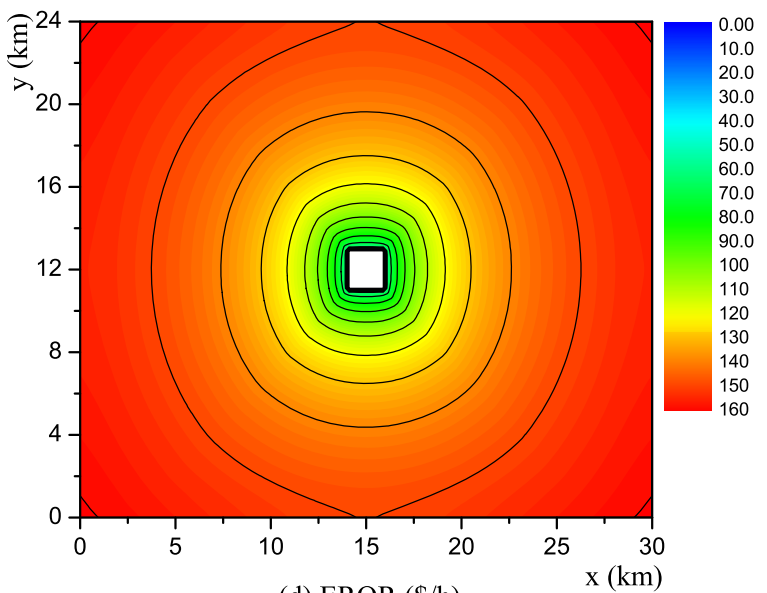

(d) EROR $(\$ / h)$

Fig. 5. The expected occupied travel time, expected vacant search time, cumulative profit, and EROR of vacant taxi drivers in each cell $(p=0.1$ and $L=15)$.
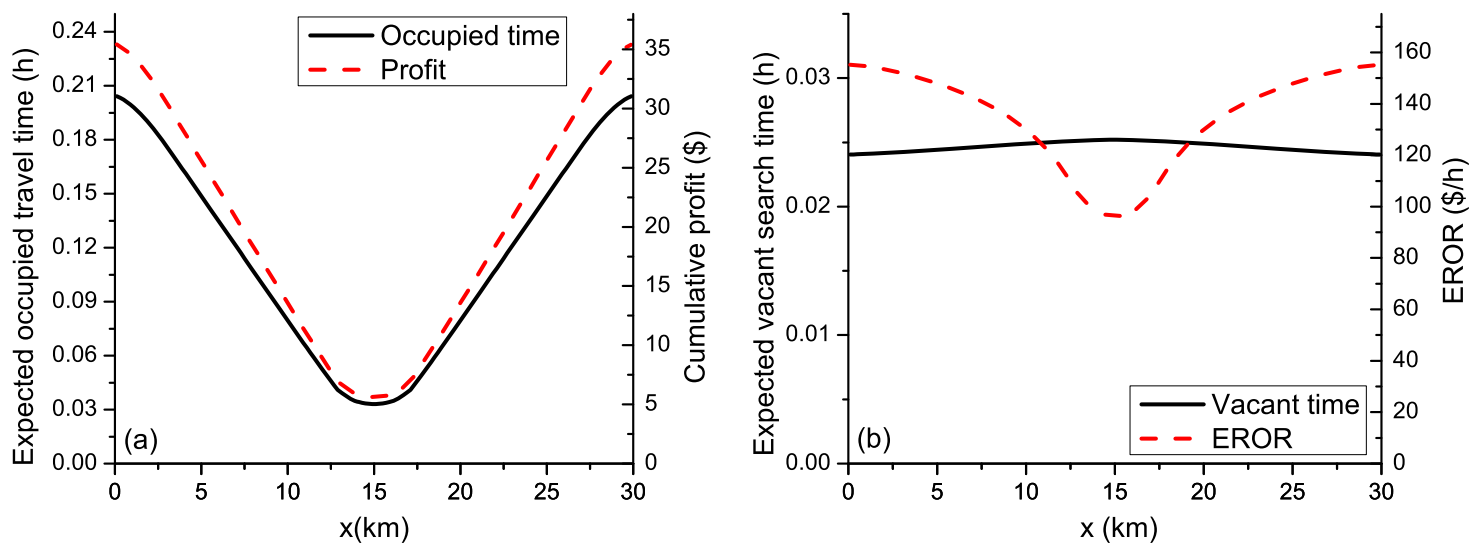

Fig. 6. The expected occupied travel time, expected vacant search time, cumulative profit, and EROR of vacant taxis at $y=10 \mathrm{~km}$. 


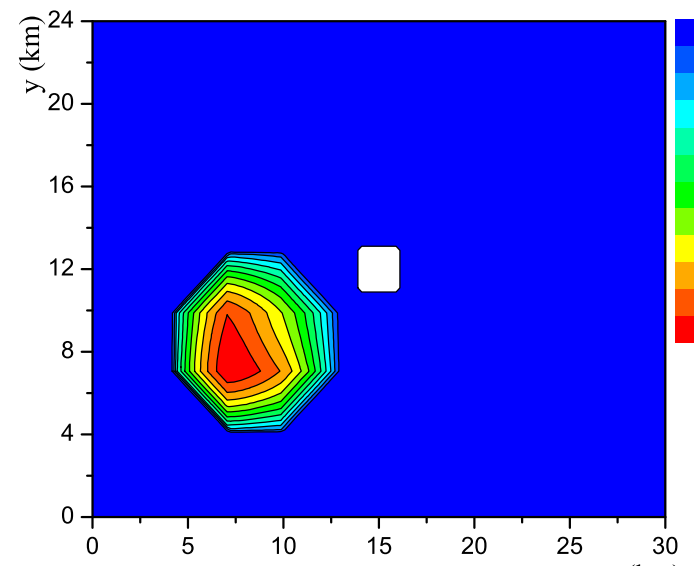

(a) Expected occupied travel time (h) $\mathrm{x}(\mathrm{km})$

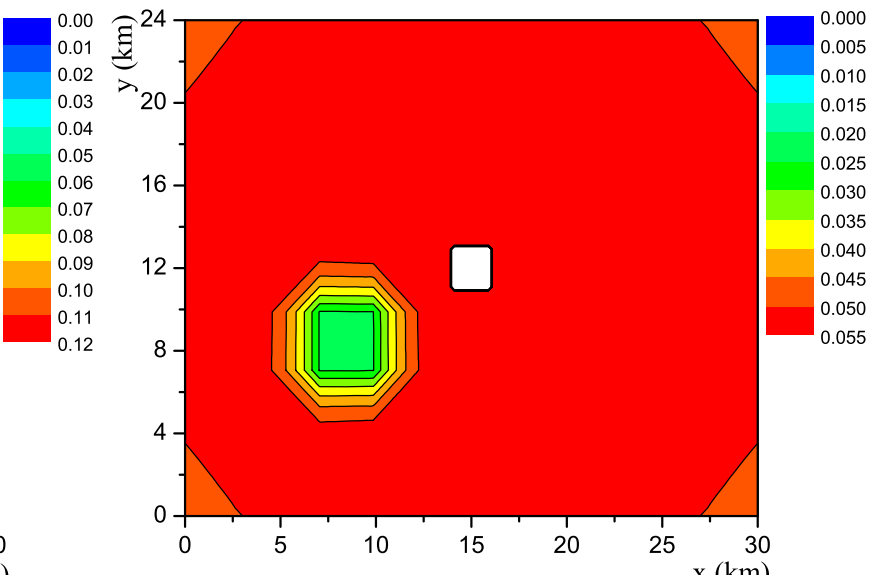

(b) Expected vacant search time (h) $\mathrm{x}(\mathrm{km})$

Fig. 7. Expected occupied travel time and expected vacant search time in Scenario 2.
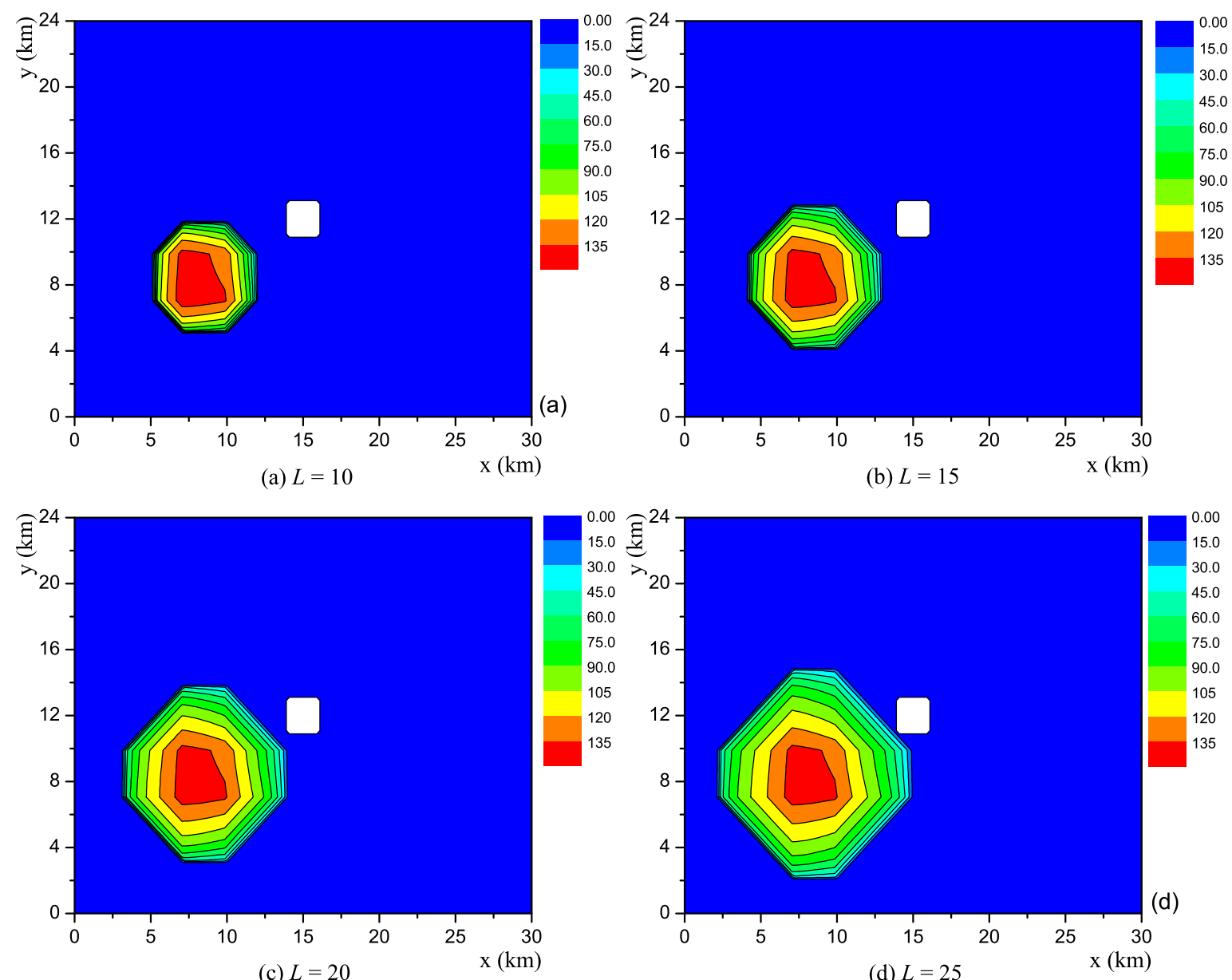

(c) $L=20$

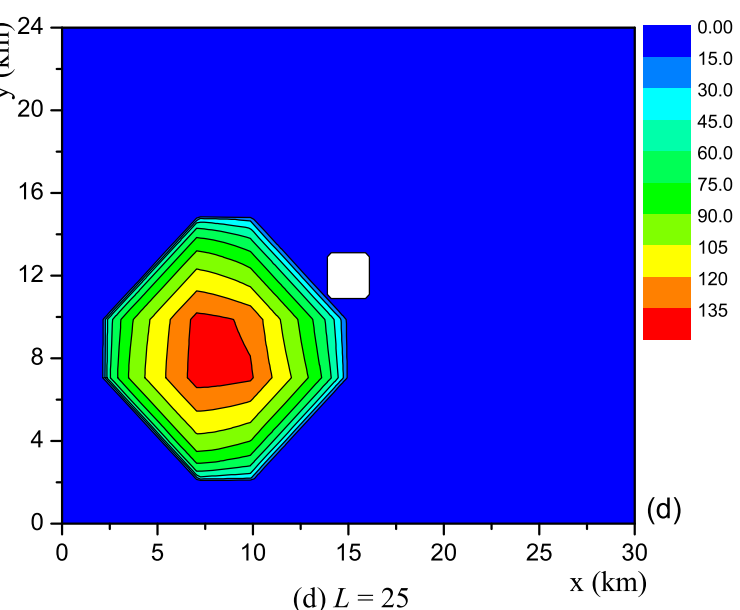

Fig. 8. The EROR of vacant taxi drivers in Scenario 2. 


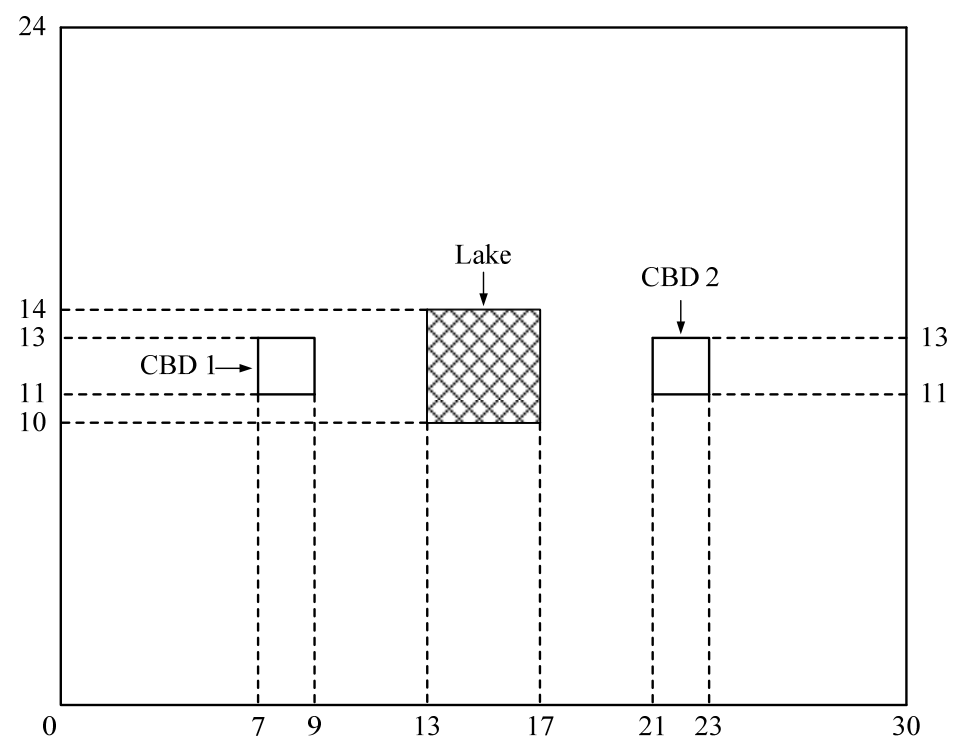

Fig. 9. The modeling domain with two CBDs and one lake.
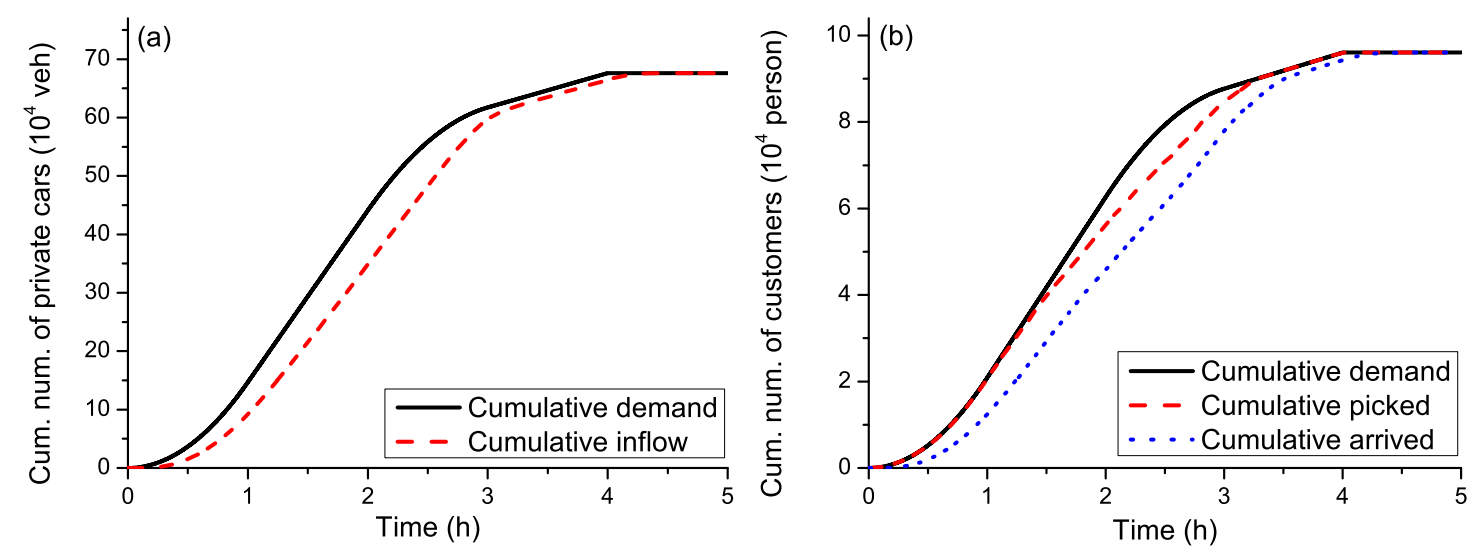

Fig. 10. The cumulative numbers of private cars and customers during the study period. 


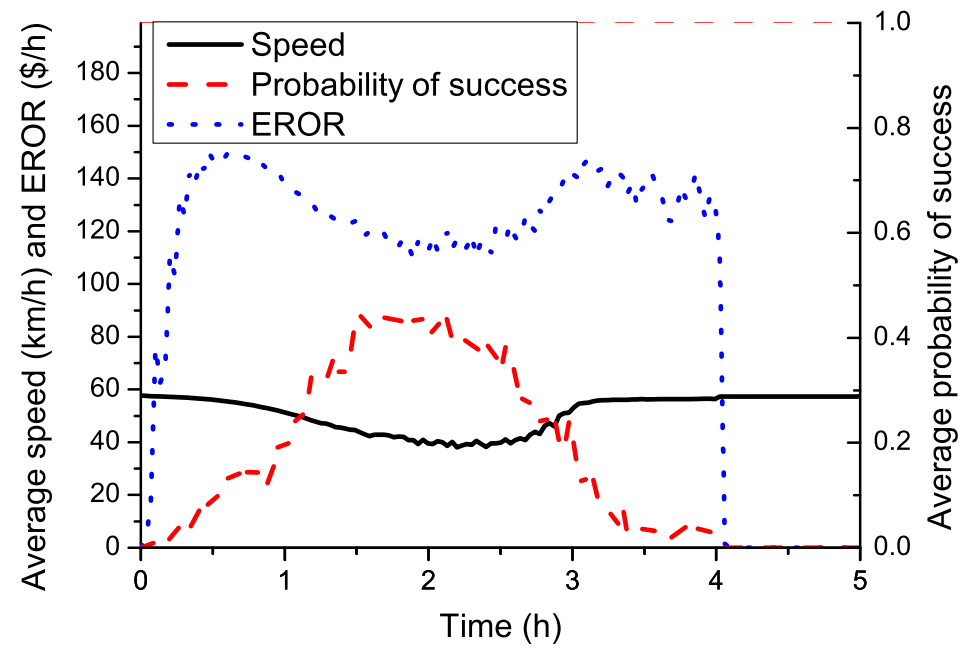

Fig. 11. The average speed, probability of success, and EROR of the whole modeling domain. 

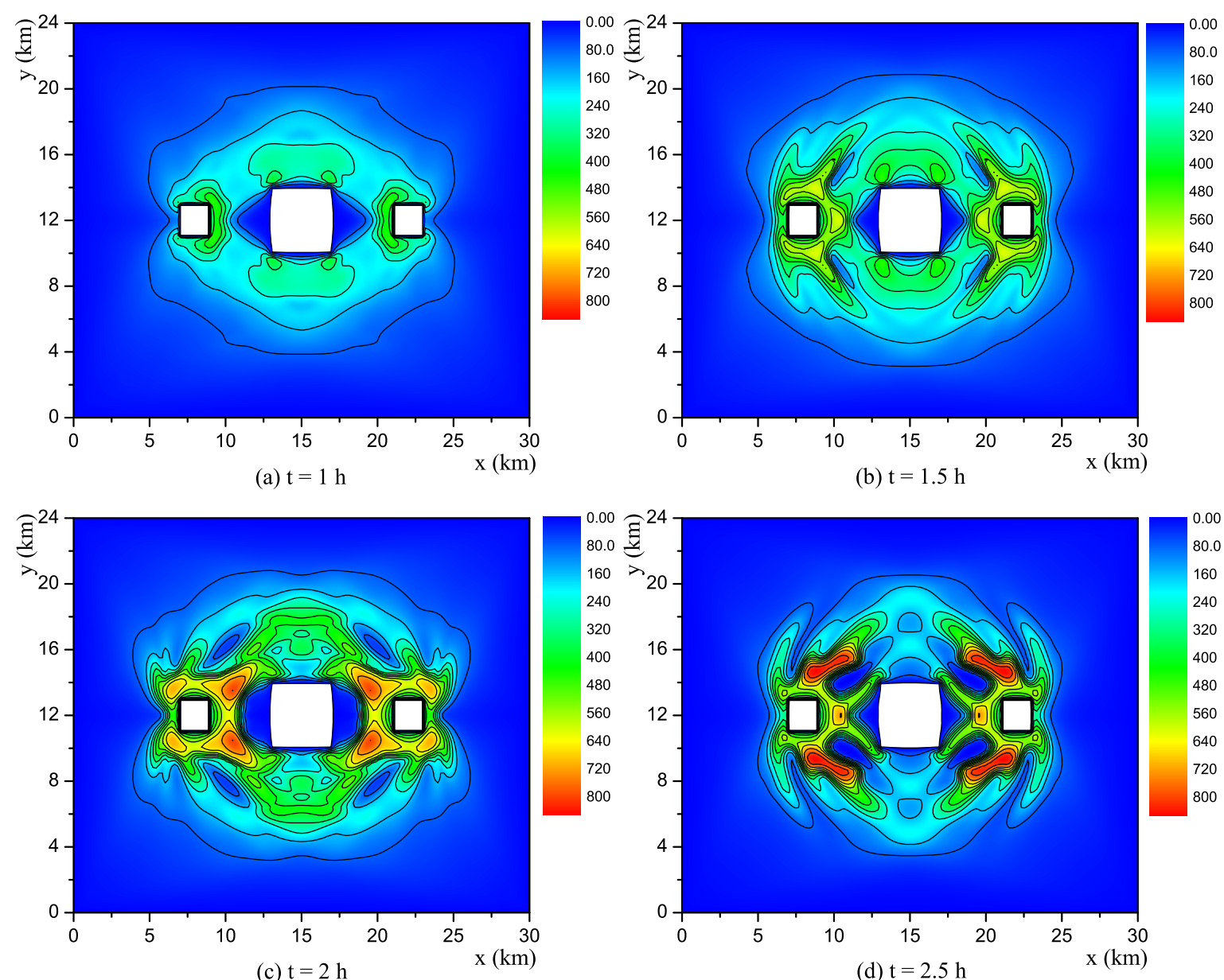

(c) $\mathrm{t}=2 \mathrm{~h}$
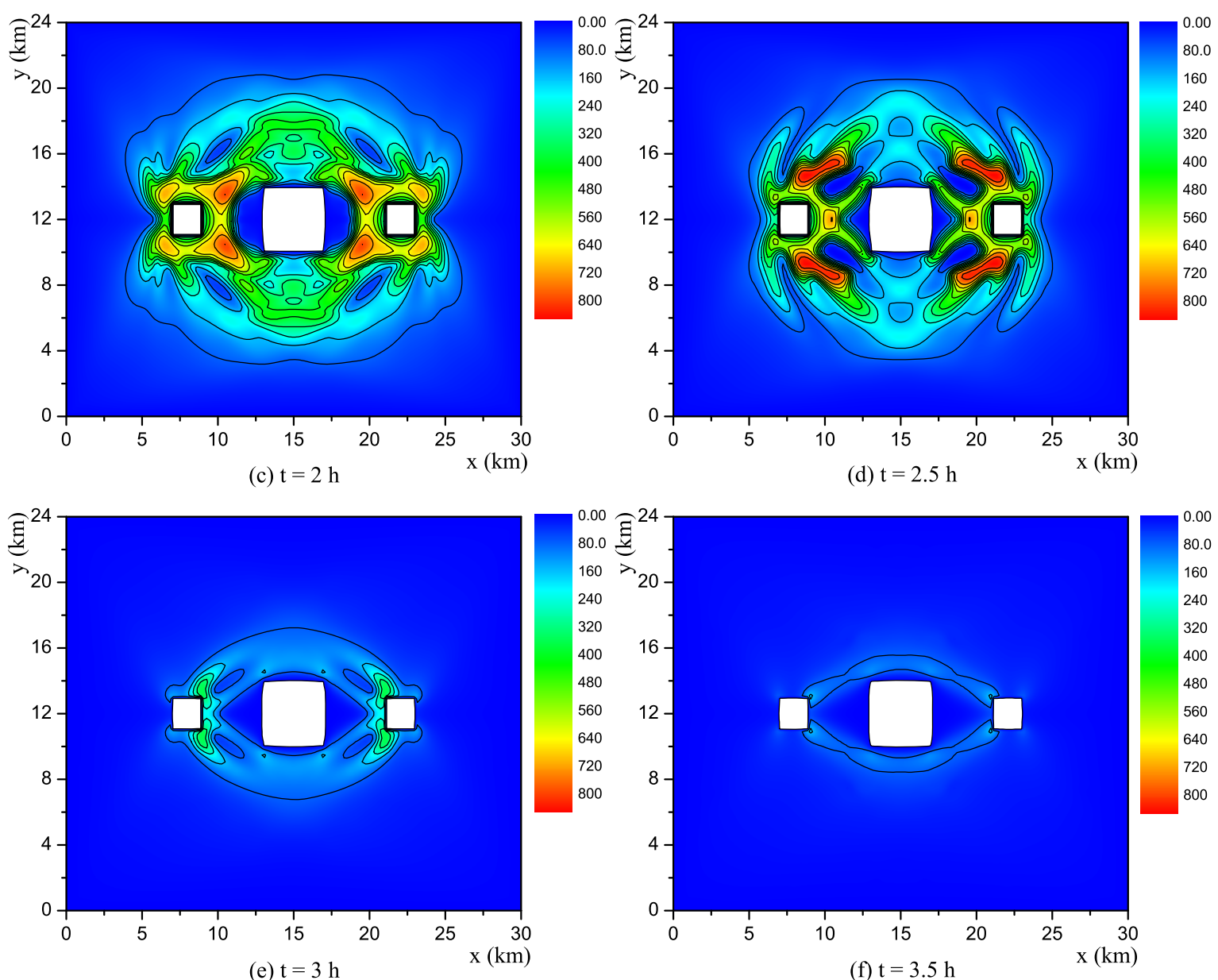

Fig. 12. The density of private cars. 

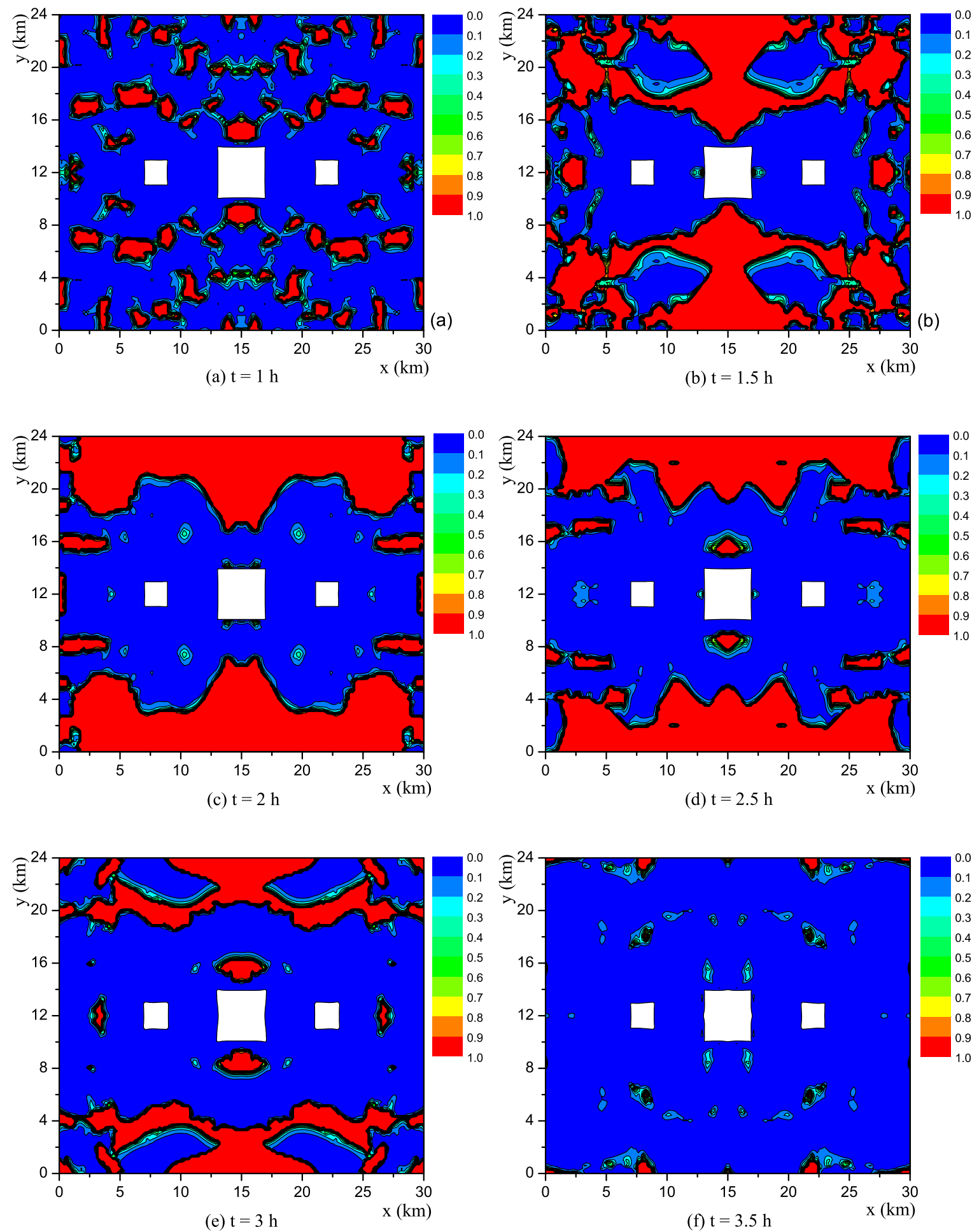

Fig. 13. The probability of success. 

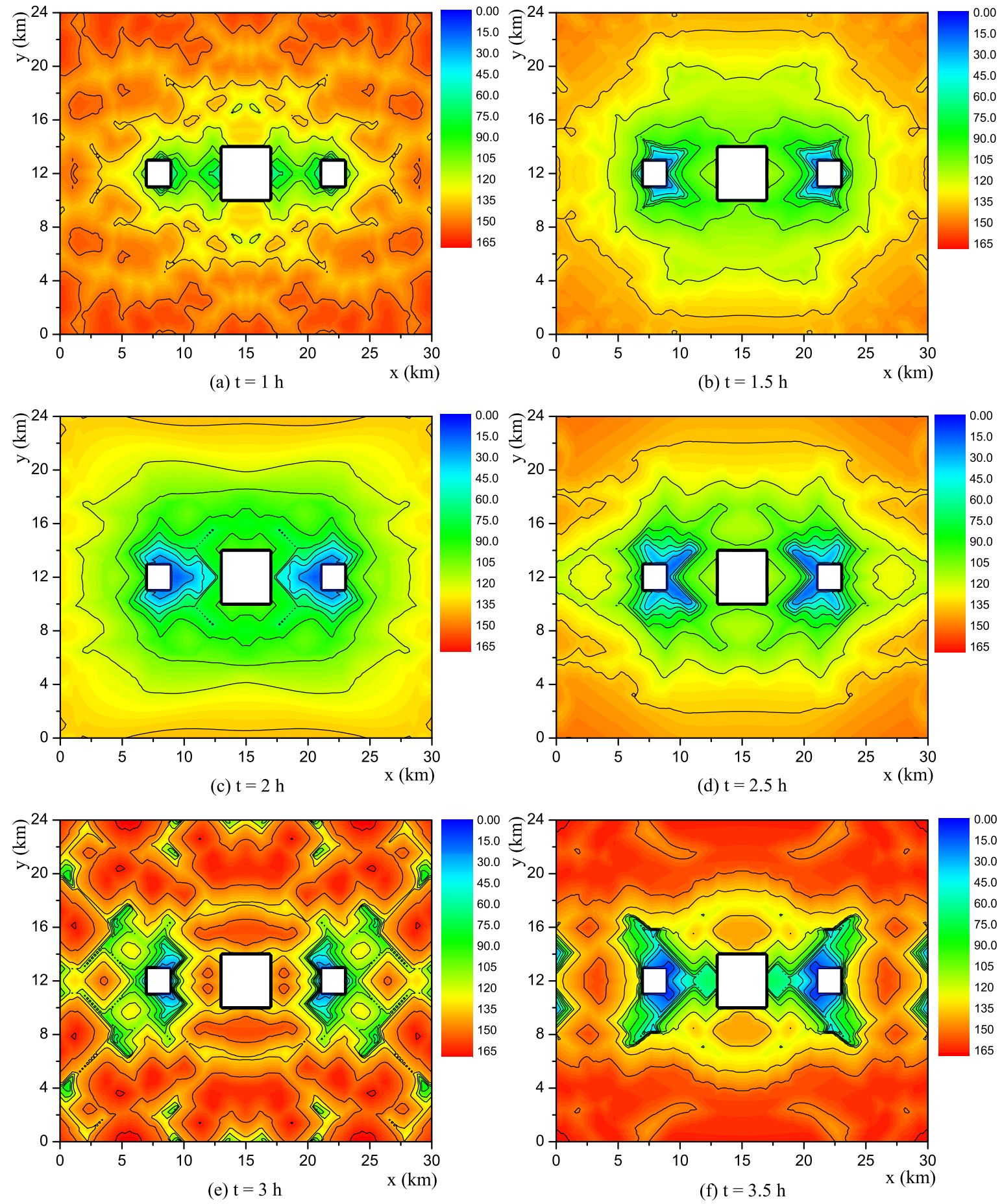

Fig. 14. The expected rate of return. 


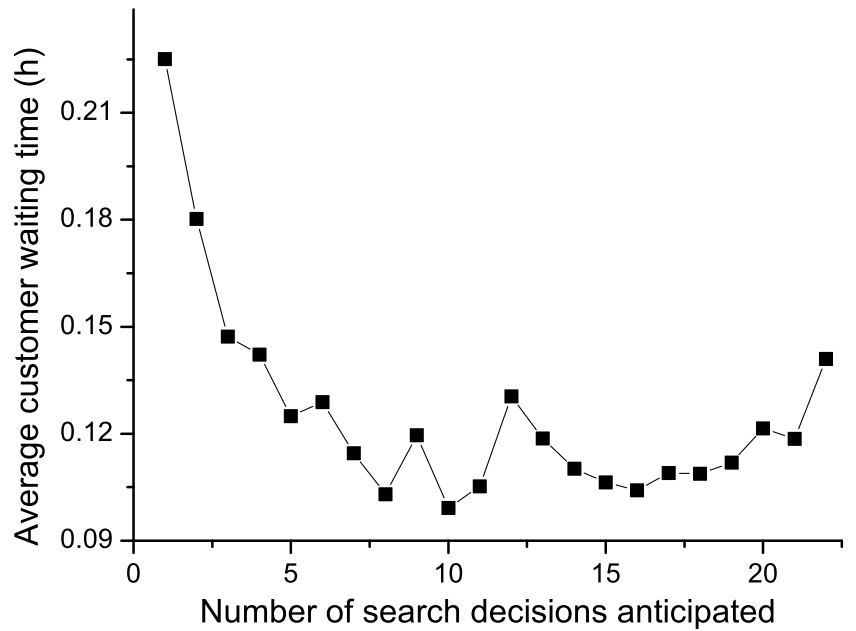

Fig. 15. The influence of the number of search decisions anticipated on the average customer waiting time.

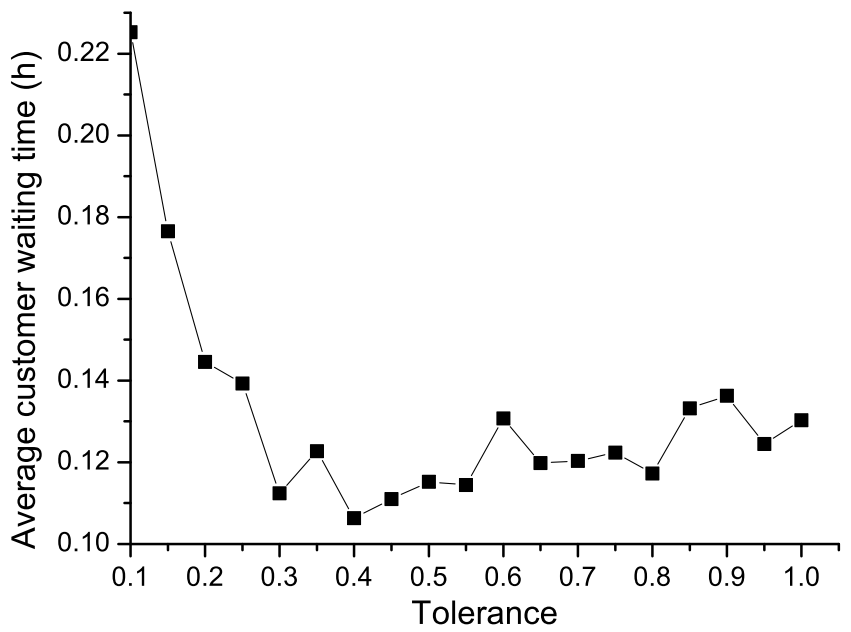

Fig. 16. The influence of tolerance on the average customer waiting time.

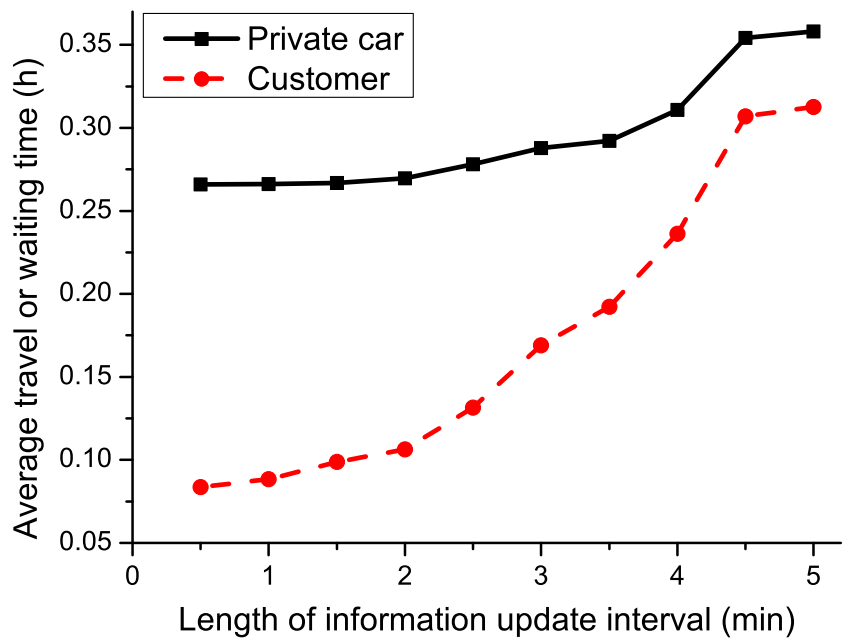

Fig. 17. The influence of the length of information update interval on the average travel time of private cars and the average customer waiting time. 


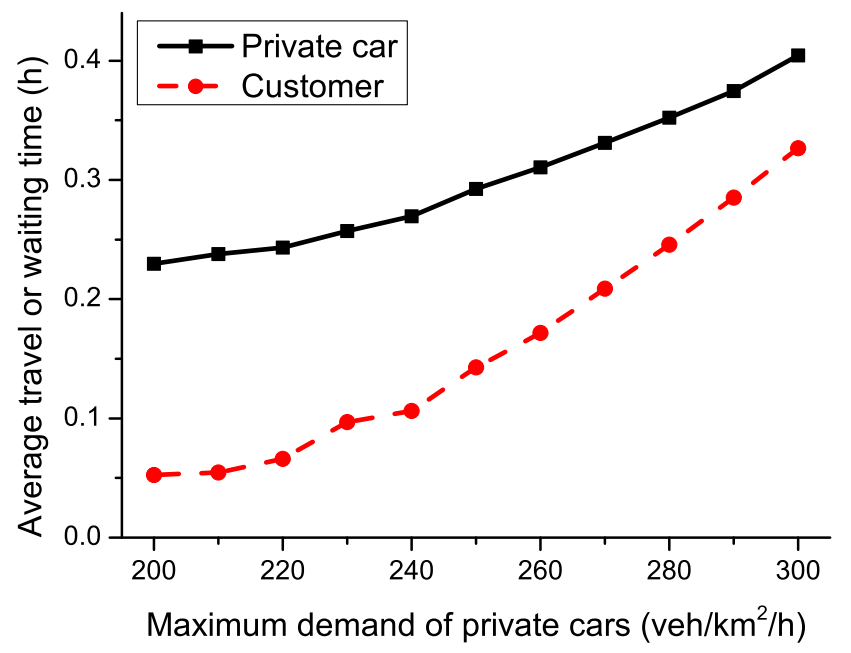

Fig. 18. The influence of private car demand on the average travel time of private cars and the average customer waiting time.

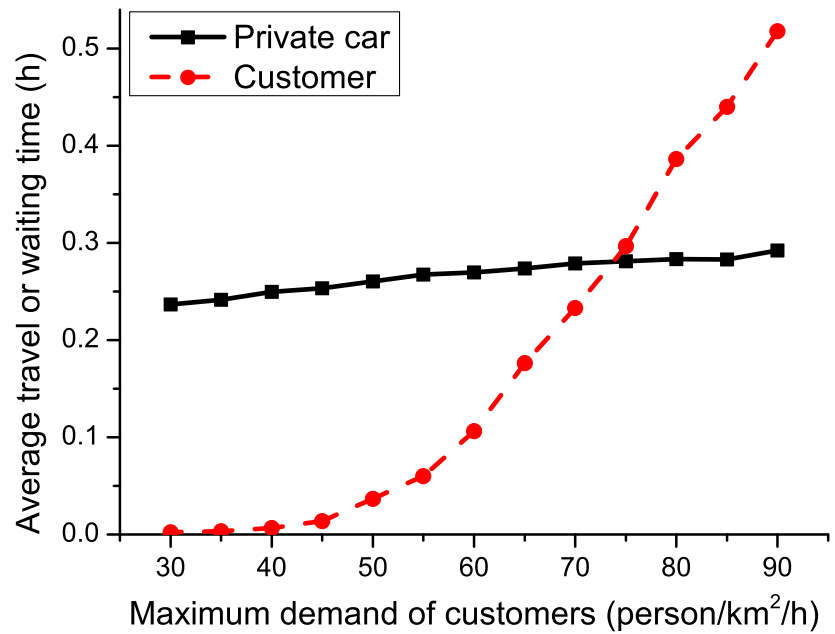

Fig. 19. The influence of customer demand on the average travel time of private cars and the average customer waiting time. 


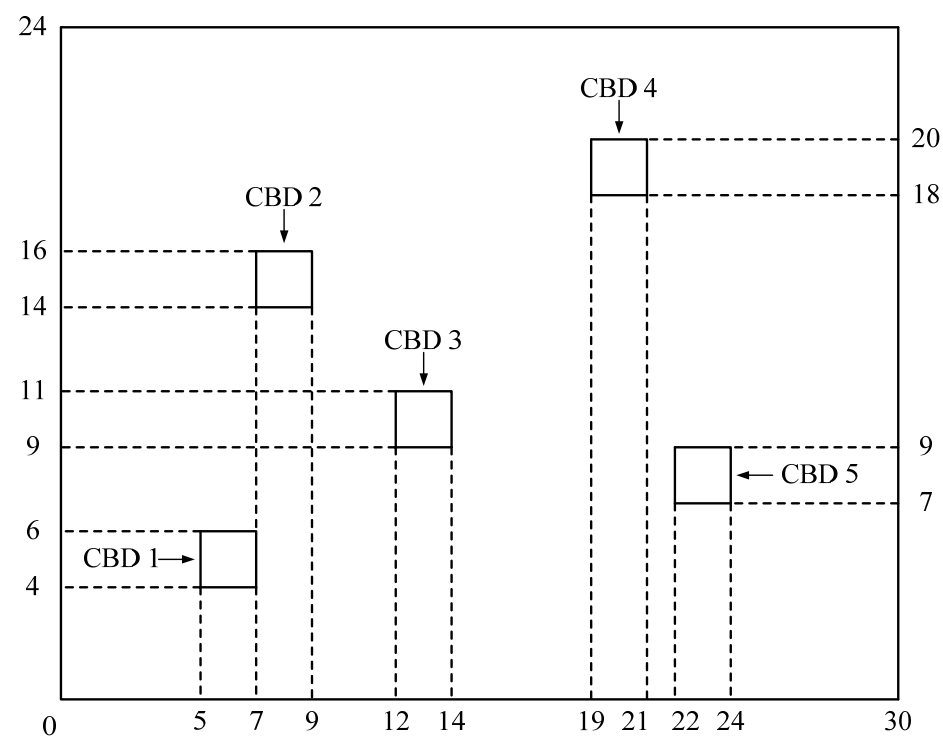

Fig. 20. The modeling domain with five CBDs.

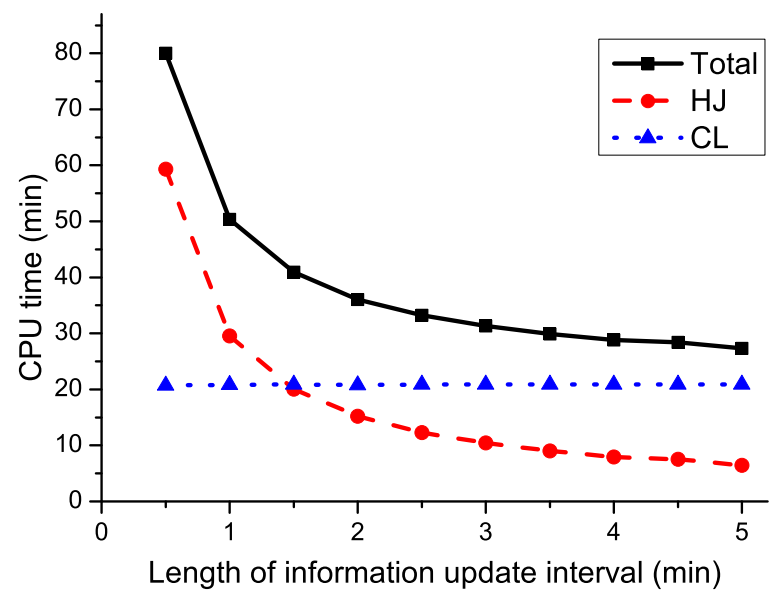

Fig. 21. The effect of length of information update interval on CPU time.

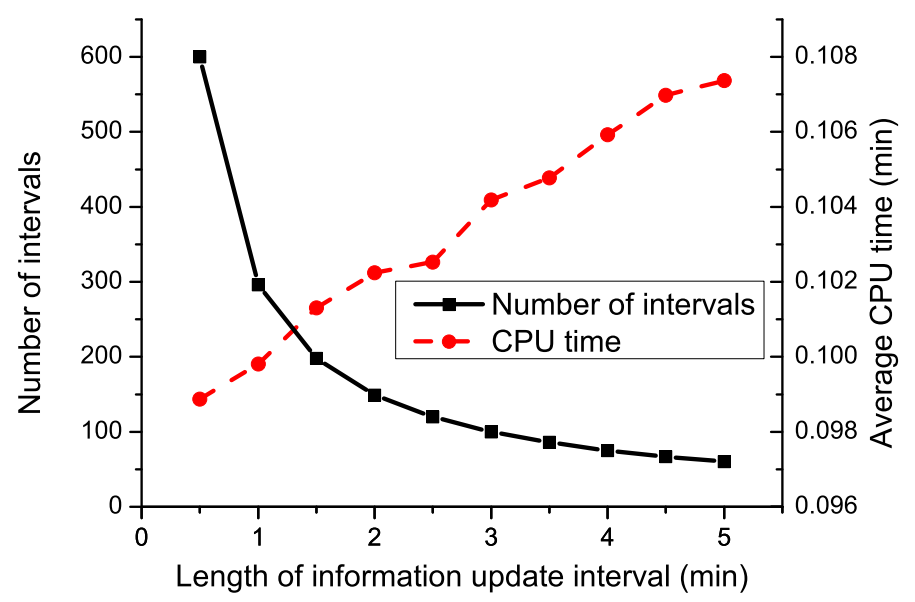

Fig. 22. The effect of the length of information update interval on the number of intervals in the modeling 
period and the average CPU time for solving HJ equations for one interval.

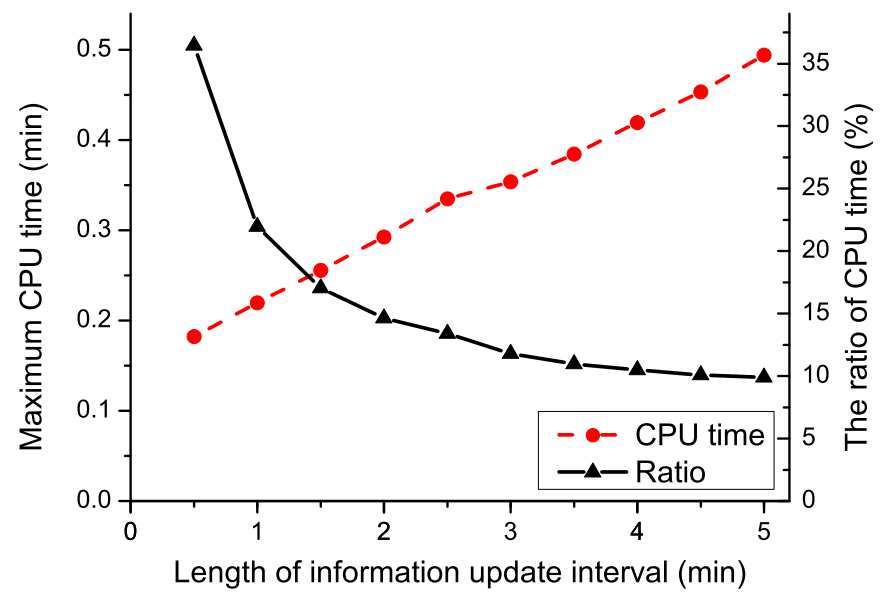

Fig. 23. The effect of the length of information update interval on the maximum CPU time for solving the whole problem for one interval, and the ratio of the CPU time to the length of information update interval.

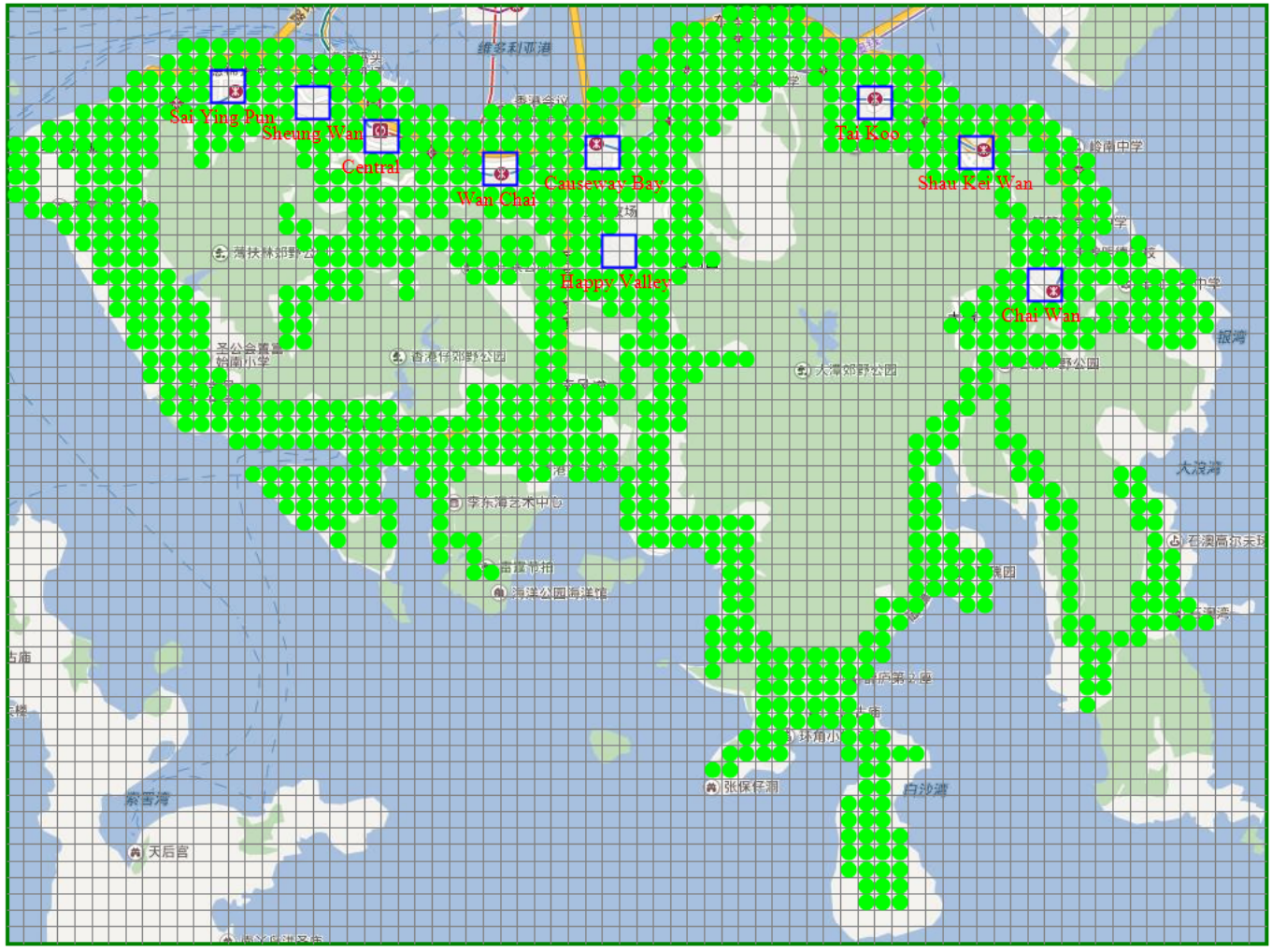

Fig. 24. The modeling domain of Hong Kong Island. 

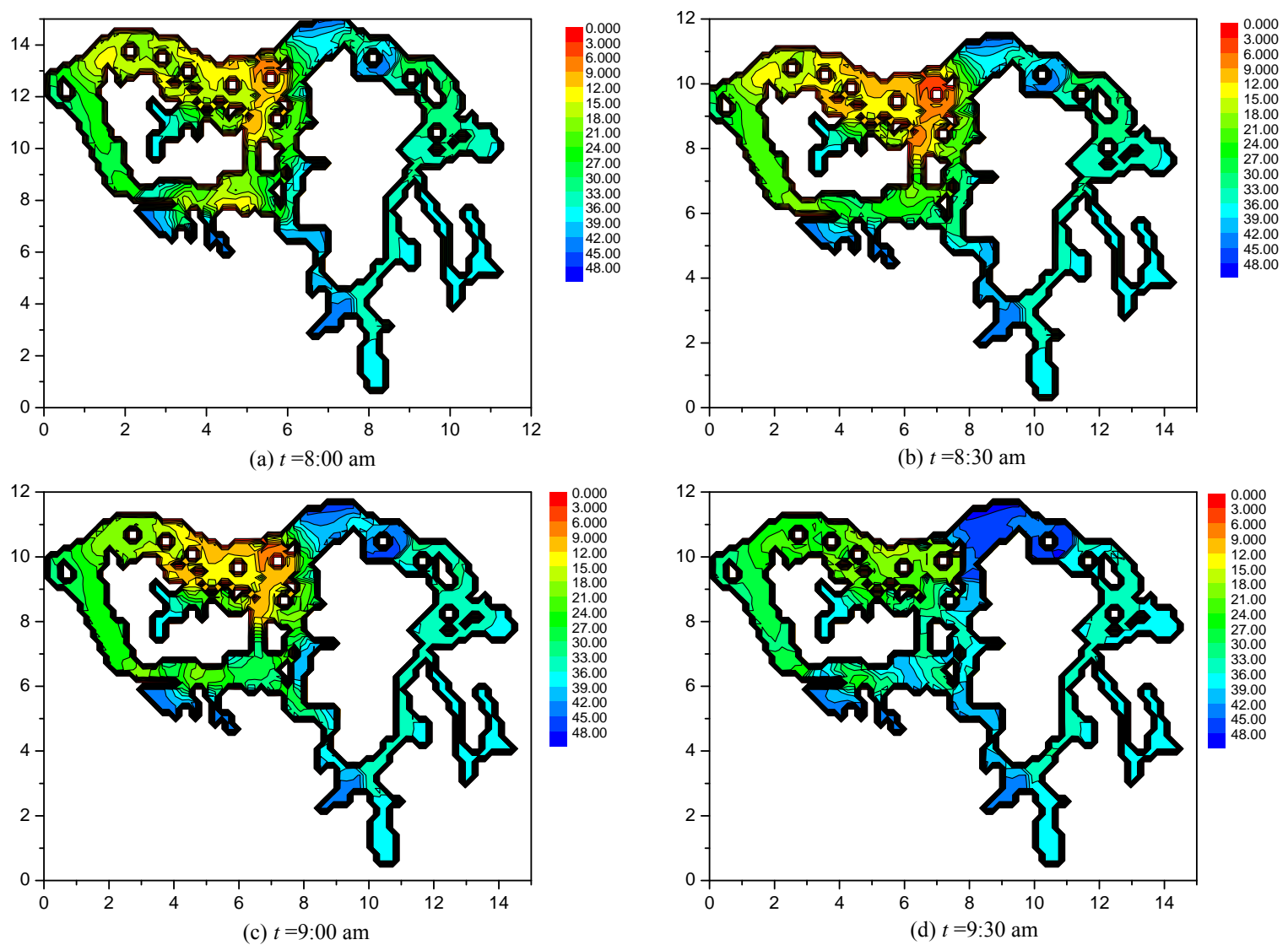

Fig. 25. The speed distribution of the modeling domain in Hong Kong Island at four different time instants. 